\title{
LA CONQUISTA DE LA INFLACIÓN: REVISIÓN Y ANÁLISIS DE LA LITERATURA RECIENTE**
}

\author{
Francisco Rosende
}

A partir de mediados de los 80 se verifica una caída importante en la inflación en las economías industrializadas, proceso que más tarde se extiende a numerosos países en desarrollo. Este proceso se produce en un contexto intelectual donde se aprecia con claridad la influencia de ideas como: "la hipótesis de la tasa natural"; las "expectativas racionales" y la "neutralidad de largo plazo" de la política monetaria. En esta perspectiva, resulta tentador suponer que la caída experimentada por la inflación en el mundo ha sido el resultado de un genuino progreso en el conocimiento adquirido por la Ciencia Económica.

A partir de una revisión de la literatura reciente sobre las causas del aumento y posterior caída de la inflación en los Estados Unidos, en este artículo se examina la validez y alcance de la visión optimista antes mencionada.

En la segunda parte del trabajo se discuten las interpretaciones que se han planteado en el caso chileno respecto a dicho proceso, a la luz de una identificación de las restricciones y objetivos que ha enfrentado la política monetaria en los últimos treinta años.

Francisco Rosende R. Decano de la Facultad de Ciencias Económicas y Administrativas, P. Universidad Católica de Chile. Investigador asociado del Centro de Estudios Públicos.

* Agradezco los valiosos comentarios de Rodrigo Vergara. También agradezco a los participantes del seminario interno del Instituto de Economía de la P. Universidad Católica de Chile.

Estudios Públicos, 109 (verano 2008). 


\section{Introducción}

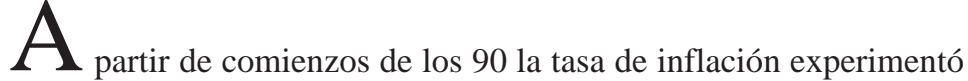
una caída importante en el mundo, al pasar de un $26,5 \%$ a un $3,8 \%$ el año 2006, como se indica en el Cuadro $N^{\circ} 1$. Una trayectoria similar se observa en el caso chileno.

CUADRO N 1-A: INFLACIÓN: GRUPOS DE PAÍSES

\begin{tabular}{|c|c|c|c|c|}
\hline & \multicolumn{4}{|c|}{ Variación promedio (\% anual) } \\
\hline & $\begin{array}{c}\text { Economía } \\
\text { mundial }\end{array}$ & $\begin{array}{c}\text { Economías } \\
\text { desarrolladas }\end{array}$ & $\begin{array}{c}\text { Economías } \\
\text { emergentes* }\end{array}$ & Chile \\
\hline 1990 & 26,5 & 5,9 & 67,1 & 26,0 \\
\hline 1991 & 21,9 & 4,9 & 53,4 & 21,8 \\
\hline 1992 & 35,9 & 3,8 & 105,6 & 15,4 \\
\hline 1993 & 34,4 & 3,1 & 100,0 & 12,7 \\
\hline 1994 & 27,8 & 2,6 & 77,4 & 11,4 \\
\hline 1995 & 14,4 & 2,6 & 34,3 & 8,2 \\
\hline 1996 & 8,5 & 2,4 & 17,9 & 7,4 \\
\hline 1997 & 5,9 & 2,1 & 11,5 & 6,1 \\
\hline 1998 & 5,4 & 1,5 & 11,2 & 5,1 \\
\hline 1999 & 5,0 & 1,4 & 10,2 & 3,3 \\
\hline 2000 & 4,2 & 2,2 & 7,0 & 3,8 \\
\hline 2001 & 4,0 & 2,1 & 6,5 & 3,6 \\
\hline 2002 & 3,3 & 1,5 & 5,7 & 2,5 \\
\hline 2003 & 3,5 & 1,8 & 5,7 & 2,8 \\
\hline 2004 & 3,6 & 2,0 & 5,4 & 1,1 \\
\hline 2005 & 3,7 & 2,3 & 5,2 & 3,1 \\
\hline 2006 & 3,6 & 2,3 & 5,1 & 3,4 \\
\hline 2007 & 3,9 & 2,1 & 5,9 & 4,4 \\
\hline
\end{tabular}

* De acuerdo a la clasificación del FMI, "Other emerging markets and developing countries”, por lo que se excluye a las economías asiáticas de reciente industrialización.

Fuente: FMI: World Economic Outlook Database 2008.

En el caso de los Estados Unidos, el que ha sido objeto de numerosos análisis en la literatura, esta caída del ritmo inflacionario se inició a comienzos de los ochenta, luego de la aplicación de un severo ajuste monetario bajo la conducción de Paul Volcker como presidente de la Reserva Federal. Dicho programa de ajuste se origina en el elevado nivel que había alcanzado la inflación tras el quiebre del sistema de Bretton Woods a fines 
CUADRO N¹-B: INFLACIÓN: GRUPO DE PAÍSES

\begin{tabular}{lrrrrr}
\hline \multicolumn{5}{c}{ Variación promedio (\% anual) } \\
\cline { 2 - 5 } & Australia & Nueva Zelanda & Estados Unidos & Brasil & México \\
\hline & & & & \\
1990 & 7,3 & 6,1 & 5,4 & $2.947,7$ & 26,7 \\
1991 & 3,2 & 2,6 & 4,2 & 477,4 & 22,7 \\
1992 & 1,0 & 1,0 & 3,0 & $1.022,5$ & 15,5 \\
1993 & 1,8 & 1,3 & 3,0 & $1.927,4$ & 9,8 \\
1994 & 1,9 & 1,8 & 2,6 & $2.075,8$ & 7,0 \\
1995 & 4,6 & 3,7 & 2,8 & 66,0 & 35,0 \\
1996 & 2,6 & 2,3 & 2,9 & 16,0 & 34,4 \\
1997 & 0,3 & 1,2 & 2,3 & 6,9 & 20,6 \\
1998 & 0,9 & 1,3 & 1,5 & 3,2 & 15,9 \\
1999 & 1,5 & $-0,1$ & 2,2 & 4,9 & 9,6 \\
2000 & 4,5 & 2,6 & 3,4 & 7,1 & 6,4 \\
2001 & 4,4 & 2,6 & 2,8 & 6,8 & 5,0 \\
2002 & 3,0 & 2,6 & 1,6 & 8,4 & 4,5 \\
2003 & 2,8 & 1,7 & 2,3 & 14,8 & 4,7 \\
2004 & 2,3 & 2,3 & 2,7 & 6,6 & 4,0 \\
2005 & 2,7 & 3,0 & 3,4 & 6,9 & 3,6 \\
2006 & 3,5 & 3,4 & 3,2 & 4,2 & 3,9 \\
$2007 *$ & 2,3 & 2,4 & 2,7 & 3,6 & \\
& & & & & \\
\hline
\end{tabular}

* Estimación Staff FMI.

Fuente: FMI: World Economic Outlook Database 2008.

de los 60, configurando lo que se ha denominado en la literatura como la "Gran Inflación”1.

Este cuadro de inflación más baja en los Estados Unidos fue acompañado de una trayectoria más estable de la tasa de crecimiento del producto, lo que es particularmente nítido a partir de mediados de los 80. Así, para algunos economistas como Mankiw (2001), Clarida, Galí y Gertler (1999) y (2000), este resultado es consecuencia de una política monetaria que, atendiendo al logro de una baja inflación, ha mostrado la flexibilidad suficiente como para atenuar los ciclos de gasto y producto, siendo Alan Greenspan un ejecutor eximio de una estrategia de política que puede describirse a través de una sencilla regla de movimiento de las tasas de interés, del tipo propuesto por John B. Taylor (1993). Para otros, como Stock y Watson (2003), la mayor estabilidad en la trayectoria del producto no se explica tanto por un mejor desempeño de la política monetaria, como por el hecho que en el período hubo menos shocks reales, lo que contribuyó decisiva-

\footnotetext{
${ }^{1}$ Por ejemplo, véase Taylor (2002).
} 
mente al resultado en cuestión. Más aun, este fue un período caracterizado por importantes ganancias de productividad, gracias a: i) la expansión del comercio mundial originada por la apertura de importantes economías, como China, India y la ex Unión Soviética y; ii) por la incorporación de los adelantos en el ámbito de la tecnología de información a los procesos productivos. Desde este punto de vista, la mayor estabilidad macroeconómica experimentada por los Estados Unidos y las restantes economías industrializadas a partir de mediados de los 80 y en los 90, habría sido consecuencia de la combinación de buenas políticas en el ámbito monetario, con un favorable entorno económico internacional.

La implementación de una decidida estrategia de control de la inflación en las economías industrializadas coincide con el apogeo de la "hipótesis de la tasa natural" propuesta por Milton Friedman ${ }^{2}$ y con la "revolución de las expectativas racionales" impulsada por las investigaciones de Robert Lucas y Tom Sargent ${ }^{3}$. Así, tras el impacto provocado por estos desarrollos, los economistas dejaron mayoritariamente de lado la hipótesis de una curva de Phillips estable, la que en este contexto prevalecería sólo en tanto no se produjesen cambios en el entorno macroeconómico que dieran origen a un ajuste en las expectativas inflacionarias del público. De este modo, la adopción del supuesto de una curva de oferta agregada de bienes y servicios inelástica a variables nominales, excepto en el corto plazo, hacía poco aconsejable enfocar la gestión de política monetaria a objetivos distintos al logro de estabilidad de precios. Así, una interpretación posible para los diferentes esfuerzos de control de la inflación que se detectan a partir de mediados de los 70, es la influencia de los desarrollos mencionados, los que definen con claridad los límites para la política monetaria.

Desde esta perspectiva, los desarrollos ocurridos en el ámbito del estudio de los ciclos económicos a partir de fines de los 60, con la publicación de la clásica conferencia de Friedman, "The Role of Monetary Policy”, habrían originado una ganancia "permanente" en la calidad de las políticas, puesto que "finalmente" los economistas habrían aprendido qué es lo que la política monetaria podría lograr y qué no. Esta interpretación es lo que Sargent (1999) denomina como "El triunfo de la hipótesis de la tasa natural".

En el contexto del ambiente intelectual mencionado, cabe destacar el importante trabajo de Kydland y Prescott, "Rules Rather than Discretion: The Inconsistency of Optimal Plans”, publicado en (1977). En este se muestra cómo una estrategia de política monetaria coherente con el logro de un

\footnotetext{
${ }^{2}$ Friedman (1968).

${ }^{3} \mathrm{Al}$ respecto véanse, por ejemplo, los artículos que aparecen en Lucas y Sargent
} (1981). 
cuadro de baja inflación puede verse modificado en la dirección contraria, esto es, en la forma de un manejo expansivo. Ello como consecuencia de la alta tasa de descuento de autoridades económicas insertas dentro del "juego político” y, por lo tanto, expuestas a la evaluación de los procesos electorales.

Sobre la base de un análisis de este tipo, más tarde se popularizó la idea de otorgar autonomía a los bancos centrales, de modo de apoyar el diseño de políticas más coherentes con la estabilidad macroeconómica en general y de precios en particular. Así, a través de un diseño institucional conducente a reducir la tasa de descuento de las autoridades monetarias, se pretendía eliminar en éstas la "tentación" de llevar a cabo políticas expansivas, una vez que las expectativas inflacionarias hubiesen alcanzado un grado razonable de confianza en la mantención de un cuadro de estabilidad macroeconómica. En la popularización que observó este esquema institucional para los bancos centrales a partir de los 90, no se puede desconocer la influencia que parece haber tenido una positiva evaluación general respecto al desempeño del Bundesbank en el período de posguerra.

En Chile este esquema institucional entró en vigencia a comienzos de los $90^{4}$, con el mandato de cautelar por la estabilidad de precios, en un país donde la inflación había sido un problema crónico. Después de casi quince años de la puesta en vigencia del mencionado esquema institucional, la economía chilena registra tasas de inflación similares a las prevalecientes en los países industrializados ${ }^{5}$, lo que ha llevado a destacar el aporte de éste, junto con el planteamiento — cada vez más explícito— por parte del Banco Central, respecto a la importancia de la meta de inflación en sus decisiones. Así, de la experiencia chilena parece desprenderse otro ejemplo favorable a la idea del "triunfo de la hipótesis de la tasa natural".

No obstante, también se han desarrollado interpretaciones algo más “incrédulas” con respecto al verdadero aporte de los desarrollos teóricos sobre el diseño de políticas, aun cuando, en rigor, éstas no constituyen en sí mismas un cuestionamiento a la validez de dichos desarrollos. Así por ejemplo, parece razonable suponer que economías con una larga tradición inflacionaria — como la misma economía chilena — no requirieron de los mencionados progresos en el ámbito de la teoría monetaria, para concluir que no existía una curva de Phillips estable, considerando el pobre desem-

${ }^{4}$ En rigor en diciembre de 1989.

${ }^{5}$ Ello no ocurrió el año 2007, lo que plantea una interrogante respecto a la interpretación que aquí se realiza del proceso de caída en la inflación. Dado que aún es temprano para establecer en qué medida este retroceso en el control de la inflación representa un cambio de tendencia en dicha variable, omitiremos un análisis específico de las causas detrás de dicho episodio. 
peño real que observaba la economía ${ }^{6}$, en el contexto de una inflación elevada y persistente, cuyos costos de eficiencia eran fácilmente perceptibles.

Esta visión más "ecléctica” de la solidez de los logros alcanzados en materia de control de la inflación destaca el desconocimiento con que habitualmente operan los bancos centrales respecto a cuáles son "los límites de velocidad" de la política monetaria, hipótesis que eventualmente puede confundirse con la intención de explotar una "curva de Phillips”. Así, período a período las autoridades estimarán el crecimiento del producto y el de la demanda por dinero, dado la información disponible. Ello de modo de conciliar el normal desenvolvimiento de la actividad económica con el cumplimiento de ciertos objetivos de inflación. No obstante, los resultados que finalmente se obtengan dependerán críticamente de la certeza de la autoridad monetaria para estimar las variables mencionadas. De este modo, la verificación de shocks de productividad que alteren la trayectoria del producto sostenible ${ }^{7}$, es probable que origine —al menos por algún tiempouna expansión monetaria incoherente con el objetivo inflacionario de la autoridad, aun cuando no hubiese sido el propósito de ésta promover un manejo monetario expansivo con el fin de estimular la actividad económica.

Desde esta perspectiva, la forma en que se realice el proceso de aprendizaje — de la autoridad y el público — respecto a la forma en que evoluciona el entorno económico, es esencial en la determinación de la forma en que se producirá la interacción entre las decisiones de política monetaria y el comportamiento de variables como la inflación y la actividad económica, en el corto plazo.

Otros análisis del proceso inflacionario en las economías industrializadas han destacado que arreglos institucionales como el de la autonomía del banco central, que buscan lograr un cuadro de estabilidad macroeconómica $^{8}$, son vulnerables a la dinámica del sistema político y a las urgencias que éste genera ${ }^{9}$.

Si bien estas interpretaciones no son esencialmente contradictorias entre sí, enfatizan aspectos diferentes de la relación entre política monetaria y ciclo económico. Así, mientras una línea de trabajo destaca la importancia del proceso de formación de expectativas y de aprendizaje, la otra enfatiza aquellos aspectos que intervienen en la determinación de la función objeti-

\footnotetext{
${ }^{6}$ Especialmente en materia de crecimiento.

${ }^{7}$ Variable esencial de establecer en cualquier ejercicio de programación monetaria.

${ }^{8}$ Este enfoque se inserta dentro de la línea de investigación impulsado por Daron Acemoglu acerca de la forma en que se construyen y evolucionan las instituciones en una economía. Al respecto, véase Acemoglu (2007).

${ }^{9}$ Sobre esta interpretación, véase Posen (1993).
} 
vo de la autoridad monetaria. En el presente artículo se revisan las interpretaciones más conocidas para explicar este proceso de caída en la inflación mundial que se registra a partir de los 90. En la primera parte el análisis se concentra en la experiencia de los EE.UU. y la literatura que este ha generado. En la segunda se examina la experiencia chilena. Finalmente se plantean las principales conclusiones que emergen de esta revisión.

\section{Marco general}

Desde que se publicó en 1977 el clásico artículo de Kydland y Prescott antes mencionado, acerca de la importancia de la estructura de incentivos que enfrenta el banco central en la definición de su estrategia de política monetaria, la búsqueda de explicaciones para los cambios en la inflación se ha organizado esencialmente sobre la base de dicho marco de análisis. Esto es, a partir de un estudio de las motivaciones — costos y beneficiosdetrás del diseño de política monetaria. De este modo, la discusión no se ha situado tanto en términos de la vieja controversia respecto a las causas últimas de la inflación, puesto que parece existir un grado importante de acuerdo en cuanto al origen monetario de ésta, sino que más bien respecto a los motivos que llevan a los bancos centrales a adoptar una política monetaria expansiva en un momento del tiempo y un programa de estabilización en otro.

Una primera aproximación al tipo de razonamientos que motivaron cambios en la trayectoria inflacionaria en las economías industrializadas en la segunda mitad del siglo pasado se puede obtener a partir del análisis de una ecuación del tipo "curva de Phillips", como la indicada en (1). De acuerdo con ésta, la inflación se determina por las expectativas construidas en el pasado respecto al nivel que esta variable alcanzaría en "t"10 y por la "brecha de producto" $(x)$. Este tipo de ecuación para la tasa de cambio de los precios se inserta dentro del marco de la tradición keynesiana que predominó con fuerza hasta comienzos de los 70. En general, su formulación supone la existencia de un esquema de competencia monopolística, lo que permite establecer una estrecha relación entre el comportamiento de los costos de producción - esencialmente los salarios- y la trayectoria de los precios de los bienes finales. Este mismo supuesto lleva a considerar la brecha de producto (" $x$ ”) como el principal determinante de la inflación, puesto que en este esquema la capacidad de ajustar precios de las empresas depende críticamente de la fase del ciclo de actividad en que se encuentre la economía.

\footnotetext{
${ }^{10}$ Las que se incorporan en los contratos salariales.
} 
En lo que se refiere a la determinación de los salarios nominales propiamente tal, se supone la existencia de contratos que resultan costosos de modificar. En el diseño de los mismos, una variable fundamental es la expectativa de inflación que tenían los individuos al momento de establecer sus contratos.

Así se tiene

$$
\pi(\mathrm{t})=\mu \mathrm{Et}-1 \pi(\mathrm{t})+\gamma x(\mathrm{t})+\varepsilon(\mathrm{t})
$$

donde

$\pi(\mathrm{t})=\mathrm{p}(\mathrm{t})-\mathrm{p}(\mathrm{t}-1)=$ la tasa de inflación

$x(\mathrm{t})=\mathrm{y}(\mathrm{t})-\mathrm{yn}(\mathrm{t})=$ brecha de producto, siendo $\mathrm{yn}(\mathrm{t})$ el producto "natural” o "potencial”.

$\varepsilon(\mathrm{t})$ es un shock aleatorio con media cero y varianza finita. Además $\mathrm{E}[\varepsilon(\mathrm{t}) \varepsilon(\mathrm{t}-\mathrm{i})]=0$.

Es importante advertir que en la definición de la estrategia óptima de política monetaria el parámetro “ $\gamma$ ” juega un papel determinante. En efecto, un elevado valor de dicho parámetro plantea un escenario desfavorable de “curva de Phillips”, puesto que cualquier manejo expansivo de demanda agregada tendrá un fuerte impacto en la inflación ${ }^{11}$. Lo contrario ocurre si se estima que el valor de " $\gamma$ " es reducido"12.

Como ha mostrado la literatura reciente en lo que se ha denominado como la "Nueva Síntesis Keynesiana"13, el modelo se completa con una ecuación que relaciona los movimientos cíclicos de la actividad con el impulso de la política monetaria, como se plantea en (2).

$$
x(\mathrm{t})=\omega x(\mathrm{t}-1)+(1-\omega) \operatorname{Et} x(\mathrm{t}+1)+\phi \mathrm{f}(\mathrm{t})+\mathrm{v}(\mathrm{t}) \quad 0<\omega<1
$$

donde:

$\mathrm{f}(\mathrm{t})$ es un indicador del impulso de política monetaria, el que en ciertos contextos se identifica con el desequilibrio en dicho mercado ${ }^{14} \mathrm{y}$ en otros con un indicador de tasa de interés real.

$v(t)$ es un shock aleatorio con media cero y varianza finita. Además $\mathrm{E}[\mathrm{v}(\mathrm{t}) \mathrm{v}(\mathrm{t}-\mathrm{j})]=0$, para todo j distinto de cero.

\footnotetext{
${ }^{11}$ En este caso nos acercamos al modelo neoclásico de precios flexibles.

12 Esto dentro del horizonte de referencia.

${ }^{13} \mathrm{Al}$ respecto véase, por ejemplo, Clarida, Galí y Gertler (1999).

${ }^{14} \mathrm{El}$ desequilibrio entre la oferta y la demanda por dinero.
} 
De la ecuación (2) se desprende que la "brecha de producto" puede escribirse como una función del manejo monetario, pasado, presente y esperado.

La función de pérdida de la autoridad, sobre la base de la cual resuelve sus acciones de políticas, se plantea en (3), adoptándose la habitual función cuadrática, donde el valor del parámetro " $\alpha$ ” es esencial para efectos de describir las preferencias de la autoridad entre "ambos males”: inflación y ciclos de actividad.

$$
L(t)=(1 / 2)\left[\alpha x(t)^{2}+\pi(t)^{2}\right]
$$

En ausencia de reglas que restrinjan el proceso de toma de decisiones del banco central, la trayectoria de la inflación dependerá esencialmente de los parámetros:

$\alpha ; \omega ; \phi ; \mu ; \gamma$.

En la literatura que examina la forma en que se configura la función de pérdida del banco central ${ }^{15}$, se ha señalado que el parámetro " $\alpha$ " de la ecuación (3) es el resultado del entorno político imperante, el que determina el grado de aversión inflacionaria de la autoridad. Ello de acuerdo con los efectos distributivos de ésta y las urgencias del gobierno de turno, las que podrían afectar el nivel de autonomía efectiva del banco central respecto de éste. Por simplicidad supondremos que dicho parámetro es constante, aunque nada esencial se modifica si se asume que puede cambiar.

El parámetro " $\omega$ " indica el grado de persistencia serial en la serie de la "brecha de producto". Así, mientras mayor sea ésta, menos favorable será el escenario para la política monetaria, en cuanto serán necesarios ajustes más enérgicos en su instrumento - $\mathrm{f}(\mathrm{t})$ — cuando se trate de reducir la inflación. Una conclusión similar se obtiene en el caso en que se incluye en la ecuación (1) un rezago de la inflación.

Los parámetros “ $\phi$ ” y “ $\gamma$ ” indican el grado de influencia de la política monetaria sobre la trayectoria del producto y de allí a la inflación, respectivamente. Así, mientras más bajos sean éstos, más intensos deberán ser los ajustes en esta política con el propósito de alcanzar un cierto resultado en materia de inflación.

${ }^{15}$ Por ejemplo, Cukierman (1992), capítulos 9 y 10, y Posen (1993). 


\section{Interpretaciones}

A partir del sencillo marco analítico expuesto se han planteado diversas interpretaciones respecto a las causas que llevaron a un fuerte aumento de la inflación en los EE.UU. durante los 70 y más tarde a un severo programa dirigido a reducirla. En la perspectiva de la ecuación (1), suele explicarse la aplicación de un manejo monetario expansivo por un período - relativamente prolongado - como una consecuencia de la estimación de un bajo valor para el parámetro " $\gamma$ ” por parte de la autoridad, a lo que se añadía un alto valor de " $\alpha$ " en la función de pérdida (3), generado por el entorno político-social. Probablemente en esta perspectiva incidió el impacto de la Gran Depresión de los años 30, lo que generó una sensibilidad muy fuerte por la estabilización de la actividad, a lo que se añadió una cierta tendencia a suponer que la etapa de expansión de la actividad y el comercio internacional que caracterizó al período de posguerra, permitía sostener un manejo más expansivo de la demanda agregada, con un bajo impacto en la inflación. Para algunos economistas esta inflación —en tanto moderada - era un "precio" razonable para facilitar el funcionamiento de la actividad productiva que requería de esta especie de lubricante.

Más tarde, los costos reales asociados a una inflación que se elevó fuertemente tras el primer shock petrolero de comienzos de los 70, habrían provocado un aumento en el peso político de los perjudicados por ésta ${ }^{16}$, impulsando la puesta en marcha de un programa de estabilización. En el contexto de las ecuaciones planteadas, ello no sólo se habría hecho sentir en el costo creciente de la inflación que muestra la función de pérdida indicada en (3), sino que, además, parece razonable añadir una eventual caída de $\alpha$ en ésta, representando un mayor rechazo del público a la inflación ${ }^{17}$.

Una interpretación algo diferente ha sido planteada por Orphanides (2002), quien ha sostenido que no hubo cambios sustanciales entre el enfoque de política monetaria seguido por la Reserva Federal en los 70 respecto a lo observado en la década siguiente. A su juicio, lo que originó la alta inflación fue una persistente subestimación de la "brecha de producto" en el primer período, lo que llevó a incubar presiones inflacionarias que más tarde se manifestaron. En esta perspectiva, si se reconstruye el diseño de

\footnotetext{
${ }^{16}$ Posen (1993) adopta esta hipótesis para explicar los cambios de conducta de la Reserva Federal, bajo un mismo marco institucional de autonomía.

${ }^{17}$ En el ejercicio planteado, donde la función de pérdida sólo tiene dos argumentos, el aumento en el coeficiente de uno de ellos — en este caso de la "brecha de producto"tácitamente implica una caída en la importancia relativa del otro.
} 
política monetaria con la información efectivamente disponible para la Reserva Federal cuando tomó sus decisiones, se encuentra que no hubo un cambio fundamental en la estrategia de política monetaria. En términos de las tradicionales "reglas de Taylor", Orphanides encuentra que la elasticidad de la tasa de interés de política monetaria respecto a la estimación de la "brecha de producto" que realiza el banco central, con la información disponible en tiempo real, no es sustancialmente diferente entre ambos períodos, contrariamente a lo planteado por el propio Taylor ${ }^{18}$.

Esta interpretación ha sido cuestionada en el reciente informe elaborado por Cecchetti et al. (2007), quienes sostienen que dicha hipótesis difícilmente puede explicar un período de alta inflación que duró más de una década. No obstante, sus estimaciones confirman el resultado obtenido por Orphanides, en cuanto a que en algunos años se observan diferencias importantes entre la estimación de la "brecha de producto" obtenida con la información efectivamente disponible a la Reserva Federal — cuando debió resolver una determinada acción de política monetaria — respecto de aquellas que se elaboraron más tarde, con información definitiva.

Para ellos —Cecchetti et al. (2007) — la inflación se origina en la búsqueda de un mayor producto real, en el contexto de la tradicional explotación de una "curva de Phillips", proceso que posteriormente no pudo revertirse debido a la percepción por parte de las autoridades de que sería necesaria una fuerte caída de la actividad para conseguir una caída importante de la inflación. Así, la percepción que el parámetro “ $\gamma$ ” de la ecuación (1), era reducido, estimuló un manejo monetario expansivo durante un período prolongado. En particular, Cecchetti et al. (2007) encuentran que la política monetaria seguida en los EE.UU. se habría hecho marcadamente expansiva en presencia de los shocks petroleros que tuvieron lugar a comienzos de los 70, lo que reflejaría una estrategia de "acomodación” de los mis$\operatorname{mos}^{19}$.

Este sesgo expansivo de la política monetaria $-\mathrm{y}$ la intensidad del mismo - lo miden a partir de las desviaciones que se detectan entre la tasa de interés de política monetaria que resulta de la aplicación de una "regla de Taylor" (ipm*), calculada bajo supuestos razonables de sus parámetros ${ }^{20}$ y la tasa que efectivamente estableció la política monetaria (ipm), como se indica en (4).

$$
d=[2+\pi+1 / 2(\pi-2)+1 / 2 x]-i p m=(i p m) *-(i p m)
$$

\footnotetext{
${ }^{18} \mathrm{Al}$ respecto véase Taylor (2002).

${ }^{19}$ Este punto es coincidente con lo señalado por Taylor (2002).

${ }^{20}$ Se adopta el supuesto de una tasa de interés real neutral de $2 \%$ y de una tasa de inflación objetivo de la Reserva Federal de la misma magnitud.
} 
Cabe señalar que la regla de Taylor — que en este contexto se identifica como la regla óptima de administración de la tasa de política monetaria- surge de la minimización de (3) sujeto a una ecuación de curva de Phillips como (1), para reemplazar posteriormente la condición de optimalidad en (2), suponiendo en este caso que

$$
f(t)=[i(t)-E(t) \pi(t+1)]
$$

donde:

i(t) es la tasa de interés de mercado, la que se relaciona directamente con los movimientos de la tasa de política monetaria.

Para De Long (1996) y Romer y Romer (2002), lo que se ha denominado como el período de “Gran Inflación” en los Estados Unidos era inevitable, puesto que no hubo consenso político para llevar a cabo un enérgico programa de estabilización, con los consecuentes costos reales que ello involucra, sino hasta que los costos de la inflación alcanzaron un nivel significativo. En la perspectiva del modelo expuesto, un valor relativamente reducido del parámetro " $\gamma$ ” junto con una dinámica ascendente de las expectativas, harían necesario un fuerte ajuste en la actividad para lograr una caída de importancia en la inflación. Como se indicó, este problema se agudiza en la medida que se supone que el valor de $\omega$ en la ecuación (2) es elevado.

En su Conferencia Nobel de 1977, Milton Friedman muestra evidencia de una "curva de Phillips" positiva para un grupo de países industrializados a partir de fines de los 60. Para Friedman, esta sería la "tercera etapa” del proceso de ajuste de la economía luego de la aplicación de un manejo monetario expansivo, cuando el alza de la inflación comienza a provocar un daño considerable en la eficiencia del proceso de asignación de recursos. En el caso específico que examina Friedman, parece incuestionable que el comportamiento de las series de inflación, desempleo y producto, se vio influido por el shock petrolero de comienzos de los 70, sin embargo antes de ello se hicieron visibles los signos de un manejo monetario expansivo en los EE.UU., creándose entonces las condiciones para la aplicación de un severo plan de ajuste monetario, a pesar de los elevados costos reales que casi inevitablemente este tendría.

\section{Aprendizaje y problemas de medición}

En un importante trabajo publicado en 1999, Sargent reexamina la relación entre la política monetaria y el ciclo económico, a través de un complejo análisis teórico en el que el problema del aprendizaje tiene un 
papel protagónico. Así, se construye un modelo en el que tanto la autoridad monetaria como el público ajustan periódicamente sus expectativas respecto a la marcha de la economía, a la luz de los antecedentes que reciben. En el caso de las autoridades, se supone que éstas diseñan su estrategia de política monetaria óptima de acuerdo con la estimación que realicen de las condiciones de oferta agregada prevalecientes en la economía. En particular, un antecedente importante dentro de este ejercicio es la estimación de la forma en que interactúan la inflación y la actividad —caracterizada por el parámetro " $\gamma$ ” de la ecuación (1). La estimación del mismo definirá el "límite de velocidad" para dicha política, definido éste como el máximo ritmo de impulso monetario compatible con el logro del objetivo inflacionario de la autoridad, o con una tasa de inflación que sea aceptable a ésta.

Por su parte, el público tratará de establecer cuáles son las prioridades de la autoridad monetaria, resumidas en su función de pérdida, a través de un análisis de sus acciones, lo que también implica estimar ecuaciones como (1).

Es importante señalar que del análisis de la experiencia de los Estados Unidos en materia de inflación, Sargent concluye que un esquema de formación de expectativas del tipo "adaptativo" es más coherente con la evidencia que un modelo tradicional de expectativas racionales que el mismo impulsó con Lucas²1, de los cuales surge la "crítica de Lucas". De hecho Sargent denomina esta segunda interpretación del ciclo inflacionario de los Estados Unidos como la "reivindicación de la evaluación econométrica de las políticas”.

Contrariamente a lo planteado por la hipótesis del "triunfo de la teoría de la tasa natural”, para Sargent los progresos alcanzados en el control de la inflación no serían permanentes, sino que estarían condicionados a la forma en que evolucione la percepción de la autoridad monetaria respecto a cuál es el mencionado "límite de velocidad” de esta política. Por su lado, el público ajustará gradualmente sus expectativas inflacionarias a la luz de la información que entregan las acciones de la autoridad y la trayectoria misma de la inflación. Este marco analítico contradice la creencia en el "triunfo de la hipótesis de la tasa natural”, en cuanto no cabría esperar que la inflación permaneciera controlada en un bajo nivel en forma indefinida, sino que lo razonable sería anticipar ciclos de inflación y actividad, en función de cómo cambien las percepciones de la autoridad respecto al entorno económico ${ }^{22}$. No obstante, es necesario precisar que en los términos en que se plantea el cuestionamiento a la hipótesis del "triunfo de la tasa natural", no se puede

\footnotetext{
${ }^{21}$ Lucas y Sargent (1981).

${ }^{22} \mathrm{El}$ "límite de velocidad” antes mencionado.
} 
concluir que ello implique reconocer la existencia de una curva de Phillips estable, por el contrario, es precisamente la inestabilidad de ésta lo que origina ciclos inflacionarios.

\section{Modelo básico}

En esencia, en el modelo desarrollado por Sargent (1999) se analiza el comportamiento de la autoridad monetaria a través de la minimización de una función de pérdida como (6), pero esta vez sujeto a una ecuación de oferta agregada del tipo neoclásico, como la expuesta en (7):

$$
\mathrm{L}=(1 / 2)\left[\alpha \mathrm{U}^{2}+\pi^{2}\right]
$$

$$
\mathrm{U}=\mathrm{Un}-\theta(\pi-\pi \mathrm{e}) .
$$

En ausencia de reglas que limiten la discrecionalidad de la autoridad monetaria, se obtendría un manejo monetario coherente con una inflación como $\pi \mathrm{d}$

(8) $\quad \pi \mathrm{d}=(\theta \alpha) \mathrm{Un}$.

De acuerdo con este esquema, la autoridad monetaria aplicaría un manejo expansivo frente a la estimación de un elevado valor del parámetro $\theta^{23}$, dado una cierta ponderación del desempeño real de la economía dentro de los objetivos de ésta, reflejados en el parámetro " $\alpha$ ".

Como se indicó, en la interpretación que realiza Sargent de los ciclos inflacionarios de los Estados Unidos tiene un papel preponderante el proceso de aprendizaje del público y también del banco central, acerca del nivel de la “tasa natural de desempleo” y el valor del parámetro $\theta$. En el análisis empírico del problema, encuentra evidencia favorable a la hipótesis de un esquema de formación de expectativas del tipo adaptativo para la inflación esperada, el que contendría algún mecanismo de corrección en el parámetro de ajuste " $\alpha$ " de la ecuación (9). Recordemos que de acuerdo a este esquema

$$
\pi \mathrm{e}(\mathrm{t})=a \pi \mathrm{e}(\mathrm{t}-1)+(1-a) \pi(\mathrm{t}-1),
$$

lo que es igual a

${ }^{23}$ Este resultado es similar al que obtienen Barro y Gordon (1983b) en el caso en que no se plantean reglas que limiten la "discrecionalidad" del banco central y en que éste tampoco apunte a lograr una tasa de desempleo inferior a la "natural". 


$$
\pi e(t)=(1-a) \sum_{i=0}^{\infty} a^{i} \pi(t-i)
$$

Así, como resultado de este proceso de corrección gradual de los errores de proyección se producirán transiciones más largas entre un punto de equilibrio con alta inflación a otro con baja ${ }^{24}$. A juicio de Sargent, esta dinámica es coherente con lo que se observó en los Estados Unidos tras el programa anti-inflacionario liderado por Paul Volcker.

De acuerdo con este modelo, mientras el público aprende acerca de las consecuencias de los cambios en la política monetaria respecto a la trayectoria de la inflación, el banco central lo hace respecto a la pendiente de la curva de oferta agregada, por lo que plantea un proceso de aprendizaje similar respecto al parámetro $\theta$. Así, lo que en términos de un modelo estático se plantea como una condición de optimalidad en la administración de la política monetaria, representada por la ecuación (8), en la realidad representa un equilibrio dinámico, en la medida en que "la tasa de inflación óptima"25 fluctuará de acuerdo con las estimaciones que la autoridad realice respecto a los parámetros que interviene. Luego, la ecuación (8) se puede reescribir como (8'), donde el asterisco indica la estimación que realiza la autoridad de la variable en cuestión, con la información disponible en el período previo a la adopción de acciones de política:

$$
\text { (8') } \quad \pi \mathrm{d}=\left(\theta^{*} \alpha\right) \mathrm{U}^{*} \mathrm{n}
$$

En la evaluación empírica del proceso de control de la inflación en los Estados Unidos durante los 80, es importante mencionar el estudio de diferentes procesos de control de la inflación que realizan Bordo et al. (2007). De acuerdo con esta investigación, el programa que lideró Paul Volcker estuvo marcado por un importante grado de incertidumbre respecto a cuáles eran los objetivos de la Reserva Federal, situación que habría hecho necesario acentuar la severidad del proceso de ajuste, tras una partida más bien "tibia" —o "gradualista"— del mismo. Como consecuencia de ello se

\footnotetext{
${ }^{24}$ Ello no necesariamente implica que este tipo de esquema de aprendizaje sea ineficiente. En definitiva, la optimalidad del proceso de descuento depende de qué esté ocurriendo en el entorno macroeconómico. Esto es, si está viéndose modificado o no. Una discusión respecto a la "racionalidad" de un esquema de expectativas adaptativas se encuentra en Muth (1960).

${ }^{25}$ Esta tasa es “óptima” desde el punto de vista del proceso de minimización de la función de pérdida que enfrenta el banco central, aunque ello no ocurre desde el punto de vista social, en tanto ésta aleja la economía de la cantidad óptima de dinero. Friedman (1969).
} 
produjo una importante contracción de la actividad y el empleo en este proceso.

El análisis empírico que realizan Bordo et al. (2007) muestra un lento proceso de ajuste en las expectativas inflacionarias del público tras la puesta en marcha de programa de estabilización a fines de los 70 , lo que se podría interpretar como evidencia favorable a la hipótesis de expectativas "adaptativas-racionales” de Sargent y/o a un esquema de oferta agregada en el que predominan fricciones que obstruyen el proceso de ajuste de precios y salarios. Sin embargo, ésta no es la única interpretación posible, puesto que dicha evidencia también es coherente con la presencia de un problema de credibilidad en la perseverancia de la disciplina monetaria que pretendía impulsar la Reserva Federal. Desde esta perspectiva, para Bordo et al., la severidad que finalmente adoptó la política monetaria se justificaba precisamente por los problemas de credibilidad que provocó la falta de energía y claridad que mostró inicialmente el "Plan Volcker”.

\section{El problema de información incompleta (Orphanides)}

La hipótesis planteada por Orphanides (2002), de que las presiones inflacionarias se habrían originado en una incorrecta estimación de la "brecha de producto" por parte de la autoridad monetaria, también puede ser expresada en términos del sencillo marco analítico expuesto. En la ecuación (10) se plantea la función de pérdida de la autoridad, cuyos argumentos son la tasa de inflación ${ }^{26}$ y las desviaciones de la tasa de desempleo respecto a una estimación de la tasa natural que realiza la autoridad, la que por simplicidad se expresa como una fracción " $\kappa$ " de la tasa verdadera, tal que $\mathrm{U}^{*} \mathrm{n}=$ $\kappa U n$.

Se asume que $0<\kappa<1$.

$$
L=(1 / 2)\left[\alpha\left(\mathrm{U}-\mathrm{k} U_{\mathrm{n}}\right)^{2}+\pi(t)^{2}\right]
$$

Esta función se minimiza sujeto a $(7)^{27}$, de donde se obtiene

(11) $\pi=(1-\kappa)(\alpha \theta) \mathrm{Un}$.

\footnotetext{
${ }^{26}$ Dado un objetivo implícito de inflación cero.

${ }^{27}$ Por simplicidad se ha omitido una mayor precisión respecto a las características del proceso de formación de expectativas del público, aunque su inclusión no cambia en lo esencial las conclusiones.
} 
Suponiendo que el objetivo de la autoridad monetaria no es forzar un mejor desempeño real de la economía, ni siquiera transitorio, sino que situar ésta en sus niveles "naturales" o "potenciales", entonces, con información perfecta $(\kappa=1)$, la tasa de inflación esperada será cero. Sin embargo, el impulso monetario - y con ello la inflación - crece con el error de estimación respecto a cuál es el verdadero equilibrio real de la economía, lo que en este contexto se expresa a través de la reducción del parámetro $\kappa$. Luego, la percepción de un mayor producto potencial -menor tasa de desempleo sostenible, de acuerdo a las condiciones reales de la economía - promoverá un manejo monetario más expansivo por parte del banco central, el que en equilibrio dará origen a una tasa de inflación como la indicada en (11).

Este escenario aparece como más probable en un contexto de caída en la productividad de la economía —episodio que alcanzó importancia en los Estados Unidos durante los 60 y 70 y que se conoció como el fenómeno de productivity slowdown - el que puede haber llevado a las autoridades monetarias a proyectar un mejor desempeño real de la economía de lo que era efectivamente sostenible. Desde luego, este problema se hace más crítico en un contexto donde las autoridades usan, en algún grado, un esquema de expectativas adaptativas para proyectar la evolución de la tasa de desempleo natural o la tasa de crecimiento sostenible del producto.

\section{Restricciones políticas a la política monetaria}

Una historia diferente de la mecánica detrás de la gestación de presiones inflacionarias consiste en suponer que las autoridades se plantean como objetivo real un nivel de la tasa de desempleo que les resulte "políticamente aceptable”, digamos $5 \%{ }^{28}$. En este contexto, la aprobación de leyes o regulaciones que conduzcan a un aumento en la tasa natural de desempleo por encima de la cifra mencionada, configurará una fuerte presión para lograr un manejo monetario más expansivo, lo que en definitiva provocará un aumento en la inflación.

Para ilustrar esta hipótesis, definiremos la máxima tasa de desempleo “políticamente sostenible" como una fracción " $\psi$ ” del desempleo efectivo. Así, la diferencia entre el nivel efectivo de la tasa natural de desempleo (Un) y el nivel objetivo de la autoridad $\left(\mathrm{U}^{*} \mathrm{n}\right)$ se puede expresar como

$$
\mathrm{Un}-\mathrm{U}^{*} \mathrm{n}=(1-\psi) \mathrm{Un} \quad \text { donde } 0<\psi<1
$$

${ }^{28}$ Esta interpretación también puede plantearse en términos de la tasa de crecimiento del producto. 
puesto que la presencia de distorsiones se expresa a través de la reducción del parámetro $\psi$, el que puede asociarse con un índice de eficiencia que está en el rango [1;0].

En general dicho parámetro evolucionará de acuerdo con el grado de restricciones que se impongan sobre el funcionamiento del mercado del trabajo y la economía en general, por lo que su nivel estará influido por shocks reales que repercutan sobre la capacidad de producción de la economía, como cambios tecnológicos o shocks de términos de intercambio.

Así, en períodos de shocks reales adversos, la autoridad monetaria se arriesgará con una política monetaria expansiva, de modo de atenuar el efecto negativo sobre el empleo y eventualmente revertirlo, lo que incubará presiones inflacionarias que en algún momento aflorarán. Es importante advertir que esta interpretación de los ciclos inflacionarios es -en alguna medida - contradictoria con la interpretación neokeynesiana de la inflación, puesto que ésta subraya el papel de la "brecha de producto"29 en dicho proceso, de modo que un escenario de debilidad económica originará una tendencia a reducir el ritmo de crecimiento de los precios, proceso que podría — desde esta perspectiva - atenuar ${ }^{30}$ el impacto de una política monetaria expansiva.

No obstante, esta interpretación neokeynesiana exigiría una reacción suficientemente ágil de la política monetaria, de modo de impedir que cuando se reviertan las condiciones adversas, que apoyaron el control de la inflación, se detone un proceso inflacionario como consecuencia del exceso de liquidez que se ha acumulado. Éste es sin duda un punto de gran interés teórico y empírico, el que excede el propósito de presente trabajo, sin embargo, se plantea una línea de análisis que es necesario evaluar dentro de la construcción de una teoría más general de los ciclos inflacionarios.

La incorporación de algún tipo de vínculos entre el desempeño real de la economía y la forma en que se enfoque la política monetaria, hacen posible plantear una explicación — coherente con la Teoría Monetaria del Ingreso Nominal - para la evidencia encontrada por Kydland y Prescott (1990) de un comportamiento anticíclico de la inflación en los EE.UU., la que interpretan como un antecedente adverso a ésta ${ }^{31}$. Así, siguiendo con el

${ }^{29}$ Es interesante mencionar que la "brecha de producto" también es incorporada en la ecuación para la tasa de crecimiento de los precios que plantea Milton Friedman en su clásico “A Theoretical Framework for Monetary Analysis" (1971). No obstante, el protagonismo de esta variable en la Teoría Monetaria del Ingreso Nominal es sustancialmente menor al que le otorga la "Nueva Síntesis Keynesiana".

${ }^{30}$ En el extremo, se podría suponer que el ciclo anula el efecto de la política monetaria expansiva.

${ }^{31}$ Y por lo tanto favorable a la Teoría Real de las Fluctuaciones. 
análisis de las restricciones políticas al manejo monetario, parece razonable pensar en escenarios en los que se empuja a la política monetaria para un manejo más expansivo, con el propósito de resolver un problema de débil crecimiento o alto desempleo, el que en último término se explica por cambios adversos en las condiciones reales de la economía. En este caso será posible encontrar un cuadro de mayor inflación con un débil desempeño real, lo que se ha denominado como "estanflación” y que se manifestó precisamente tras el primer shock petrolero — a comienzos de los 70 — cuando la Fed reaccionó al mismo con un manejo expansivo. Esta interpretación es coherente con los resultados obtenidos por Taylor (2002) y Cecchetti et al. (2007), de acuerdo con los cuales la inflación se aceleró en los Estados Unidos tras la aplicación de una política monetaria expansiva, con el propósito de amortiguar los efectos del "primer shock" de precios del petróleo que ocurrió a comienzos de los $70^{32}$.

En la misma línea de argumentación antes planteada, la presencia de shocks positivos de productividad daría espacio para un ciclo "virtuoso" de mejor desempeño real de la economía e inflación más baja, en el que se confunde la influencia de estos shocks favorables con la destreza de las autoridades monetarias para llevar la economía hacia un escenario de estabilidad que apoye el crecimiento. Esta historia es coherente con el cuadro de mayor estabilidad, baja inflación y abundante oferta de crédito que caracterizó a la economía de los EE.UU. a partir de mediados de los 80 y hasta mediados de la presente década, en el contexto de la muy celebrada gestión de Alan Greenspan ${ }^{33}$ a la cabeza de la Reserva Federal.

Esta interpretación de los ciclos inflacionarios de las economías industrializadas se ubica en un punto intermedio entre los desarrollos anteriores del propio Orphanides y la hipótesis del aprendizaje por parte de las autoridades que ha planteado Sargent. Así, la trayectoria del impulso monetario a la economía — resumido en la variable " $\pi$ " — sería resultado del proceso de aprendizaje de la autoridad respecto al nivel de los parámetros “ $\kappa$ ” $\mathrm{y}$ “ $\theta$ ”, además de la influencia de los cambios en las condiciones reales de la economía en éstos.

${ }^{32} \mathrm{Si}$ bien diversos estudios han subrayado el hecho que la oferta monetaria había crecido fuertemente en la segunda mitad de los 60, hasta culminar con la crisis del sistema monetario gestado en Bretton Woods, este proceso se habría agudizado como resultado de las acciones de la Reserva Federal orientadas a mitigar los efectos reales del shock petrolero.

33 Dos interpretaciones respecto al desempeño de la política monetaria en los Estados Unidos a partir de los 80, en un contexto donde los indicadores de estabilidad macroeconómica muestran progresos importantes, se encuentran en Mankiw (2001) y Stock y Watson (2003). 
La forma en que interactúan el desempeño real de la economía y la política monetaria es examinada por Ireland (1999) para el caso de los Estados Unidos. De acuerdo a los resultados que se obtienen en dicho estudio, la caída experimentada por la inflación a partir de mediados de los 80 se explicaría por la presencia de shocks reales favorables, que al reducir la tasa natural de desempleo y elevar el crecimiento del producto, disminuyeron las presiones para la aplicación de una política monetaria expansiva.

En una perspectiva más amplia de análisis de las fuerzas que intervienen en la configuración de la política monetaria, Posen (1993) destaca la influencia del marco político en el que se desenvuelve dicha política. Ello llevaría a evaluar el peso relativo de quienes ganan y de quienes pierden con la inflación para comprender la dinámica de esta variable. Así, para Posen, en algunas economías en las que pareciera que esquemas institucionales, como el otorgamiento de autonomía al banco central, han resuelto el problema del "sesgo inflacionario", en realidad sólo reflejan una estructura de fuerzas políticas que sustenta dicho resultado, lo que podría evolucionar en el tiempo. De acuerdo con este enfoque, la forma en que determinados diseños institucionales impacten sobre la economía —en el caso planteado, la autonomía del banco central sobre la inflación- es esencialmente endógeno al entorno político existente, lo que plantea una perspectiva más ecléctica respecto al efecto de dichos arreglos. De este modo, la fiereza con la que el Bundesbank abordó la búsqueda de la estabilidad de precios en Alemania en el período de posguerra, sólo se entiende una vez que se reconoce el traumático efecto que provocó en este país la hiperinflación de comienzos del siglo pasado. Por otro lado, la traumática experiencia de la Gran Depresión en los Estados Unidos, seguida por las dificultades que ocasionó la Segunda Guerra Mundial en la economía, habrían hecho políticamente difícil sostener un manejo monetario más restrictivo en esta economía. Al menos hasta que los costos de la inflación no fuesen suficientemente altos.

En todo caso, de lo expuesto no parece razonable desprender que exista evidencia suficiente como para desestimar la contribución que hacen a la estabilidad económica de un país arreglos institucionales como el que otorga autonomía al banco central ${ }^{34}$.

\section{Algo más de evidencia: Tareas para la agenda de investigación}

Un resultado interesante que emerge de la literatura reciente se refiere al proceso estocástico del cambio en la inflación. En efecto, Cecchetti et

\footnotetext{
34 Argumentos a favor del aporte de esta teoría se encuentran en Cukierman, Webb y Neyapti (1993).
} 
al. (2007) encuentran que la autocorrelación de primer orden en la serie de cambio en la inflación es negativa en todo el horizonte de análisis, el que se inicia en los 60 y termina el año 2006. No obstante, este rasgo estadístico se acentúa en forma importante a partir de fines de los 60 y hasta comienzos de los 80 —aunque siempre en el plano de los números negativos- para caer fuertemente luego.

Dado que la autocorrelación negativa implica que un aumento en la inflación es seguido por una caída de ésta, resulta difícil explicar este resultado en el contexto de modelos en los que la inflación tiene un importante grado de inercia. Si reformulamos la ecuación (1) de modo de incorporar explícitamente el tema de la inercia inflacionaria, como se indica en (1'), lo que indican los resultados obtenidos por Cecchetti et al. (2007) es que el parámetro $\lambda$ debe ser bajo, lo que implica un enfoque de determinación de precios y salarios esencialmente del tipo forward-looking. Cabe recordar que un esquema de contratos salariales del tipo backward looking $^{35}$ — como situación predominante en la economía - tiende a "arrastrar" el efecto de aquellas perturbaciones que elevaron la inflación en algún momento, dando origen a una correlación serial positiva de los cambios de la inflación y no negativa:

$$
\pi(\mathrm{t})=\lambda \pi(\mathrm{t}-1)+(1-\lambda) \mathrm{E} t \pi(\mathrm{t}+1)+\gamma \mathrm{x}(\mathrm{t})+\varepsilon(\mathrm{t})
$$

La presencia de una autocorrelación negativa de los cambios de la inflación —especialmente en el período en que ésta fue baja— lleva a suponer la predominancia de shocks reales sobre la trayectoria de los precios, puesto que en el evento que predominan los shocks de demanda cabría esperar una autocorrelación positiva. Esto es, cuando un factor de demanda —política monetaria, por ejemplo—impulsa al alza la inflación, sus efectos son más prolongados que ante shocks reales, donde cabe suponer que el efecto al alza de un período se revierte con uno a la baja más adelante. Luego, la evidencia que encuentran Cecchetti et al. (2007) para las economías del G7 a partir de los 80, es coherente con una política monetaria muy bien calibrada y certera —además de creíble—, por lo que no arrastra errores en el tiempo.

Un segundo resultado empírico que destaca de la investigación de Cecchetti et al. (2007) se refiere a la poca capacidad predictiva que muestran los diferentes indicadores de expectativas de inflación en el período de estabilidad. Más aún, encuentran que estos responden rezagadamente a los

${ }^{35}$ Como supone habitualmente la teoría neokeynesiana. Por ejemplo, véase Taylor (1979) y Fuhrer y Moore (1995). 
desarrollos de la inflación. "Los resultados sugieren que durante el período de la Gran Inflación se podía recurrir a las expectativas para pronosticar la tendencia de largo plazo de la inflación. Sin embargo, ese poder predictivo se ha deteriorado substancialmente en el período más reciente"36.

A lo anterior añaden: "En consecuencia, las autoridades económicas debieran evitar entusiasmarse mucho con la idea de considerar las expectativas de inflación como un indicador de la credibilidad de su política, ya que es más probable que éstas sigan, más que lideren, la trayectoria de la inflación, que es el objetivo primario de la política monetaria”37.

La verificación de un período de baja inflación en numerosas economías durante la última década parece haber configurado un cuadro de estabilidad en las expectativas inflacionarias y confianza en cuanto a que éste se prolongará en el futuro. Ello ha llevado a una cierta inelasticidad de las expectativas a los movimientos de variables que teóricamente son las relevantes para explicar los movimientos de la trayectoria inflacionaria, como los agregados monetarios y los movimientos del crédito. De hecho la evidencia reciente para numerosas economías — incluyendo la chilena - encuentran una baja relación entre los movimientos del dinero y la inflación en la última década ${ }^{38}$.

Si bien quedan muchas preguntas abiertas respecto a la forma en que se relacionan las variables macroeconómicas en el corto plazo, parece poco prudente inferir conclusiones fuertes en torno a las causalidades envueltas, a partir de un escenario donde la variabilidad y nivel que muestran algunas de las variables consideradas, como la inflación, es bajo. Por otro lado, parece necesario un mayor análisis respecto a la forma en que se construyen las expectativas inflacionarias y su relación con las acciones de la autoridad, en la línea planteada por la hipótesis de expectativas racionales. Sin embargo, en un contexto donde el riesgo inflacionario es bajo y por lo mismo también lo es el beneficio de gastar recursos en informarse respecto a la trayectoria futura de esta variable, parece razonable esperar que los indicadores de expectativas muestren una menor sensibilidad frente a la

36 "The results suggest that, during the period of the Great Inflation, expectations could be used to forecast the properties of the long-term inflation trend. However, for the most recent period, that forecasting power has degraded substantially", Cecchetti et al. (2007), p. 51.

37 "Consequently, policymakers should take care not to become overly enamored of inflation expectations as a measure of their policy credibility, as these expectations seem more likely to follow than lead the measures of inflation that are the primary objectives of monetary policy", Cecchetti et al. (2007), p. 51.

${ }^{38}$ Por ejemplo, véase García y Valdés (2003) y De Gregorio (2003). 
información que reciben los mercados, contrariamente a lo que ocurre en episodios de alta inflación o inestabilidad. En todo caso, parece evidente que de la investigación empírica reciente han surgido una serie de preguntas que será necesario abordar en la investigación futura.

\section{La conquista de la inflación en Chile}

\subsection{Panorama general}

La economía chilena muestra una larga historia inflacionaria, la que alcanza sus puntos más altos en la década del setenta, coincidentemente con lo que se ha denominado el período de la "Gran Inflación” en las economías industrializadas. A partir de comienzos de los 50 la inflación observa un aumento respecto a las décadas previas, al mismo tiempo que se incrementa la volatilidad de ésta, pasando de tasas relativamente bajas a tasas elevadas en un corto período. Normalmente ello se originó en episodios donde la fijación del tipo de cambio nominal era un ingrediente importante dentro de un programa de control de la inflación, el que por alguna causa ${ }^{39}$ terminó con una fuerte devaluación y la consecuente alza en el ritmo de crecimiento de los precios.

A partir de la mitad de los 50 , se observa que prácticamente todos los gobiernos intentaron llevar a cabo un plan de estabilización de precios, el que finalmente fue abandonado, retornándose en pocos meses a tasas de inflación elevadas. Este proceso se agudizó a comienzos de los 70, como consecuencia de la implementación de una agresiva expansión monetaria y fiscal, la que difícilmente podría explicarse en el contexto de modelos como los expuestos en la sección previa, dado la magnitud de la misma. Así, en el año 1973 el déficit del sector público no financiero alcanzó un 30,5\% del PIB, mientras que la "inflación oficial”"40 llegó a 508,1\%.

Tras la caída del gobierno socialista de Salvador Allende, lo que parecía perfilarse como un programa de estabilización relativamente "gradualista" se transformó en un plan de shock el año siguiente, cuando una violenta caída de los términos de intercambio hizo necesario aplicar un severo ajuste fiscal y monetario. Así, mientras el año 1974 el déficit del sector público no financiero alcanzó a un 5,4\% del PIB, éste se redujo a un $2 \%$ el

${ }^{39}$ El deterioro de los términos de intercambio y la incapacidad para sostener un manejo disciplinado de las finanzas públicas aparecen como las más frecuentes.

${ }^{40}$ Esa calificación es importante, considerando las importantes brechas que se observaban entre los precios prevalecientes en el "mercado negro" y los del "mercado oficial”. 
año siguiente, en el contexto de una caída de 46,3\% de los términos de intercambio y de 13,3\% en el PIB real. En 1976 se verificó un superávit de $4 \%$ en el sector público no financiero, lo que deja de manifiesto la severidad del proceso de ajuste fiscal realizado.

Por otro lado, la tasa de crecimiento del dinero (M1) pasó de un $362,9 \%$ promedio en 1973 a un 231,2\% en 1974 y 257,17\% en 1975, para caer a $189,4 \%$ en $1976^{41}$. Estas cifras confirman que el ajuste macroeconómico de la economía chilena de mediados de los 70 atravesó por un severo shock fiscal, el que no tuvo una manifestación igualmente drástica en la política monetaria. Una posible explicación de ello puede ser la verificación de reiteradas dificultades en el incipiente mercado financiero, las que obligaron al Banco Central a sostener un continuo flujo de liquidez para evitar el colapso del mismo ${ }^{42}$. Otra causa fue el deliberado propósito de la autoridad monetaria por acumular reservas internacionales, a través de una combinación de política cambiaria y crediticia coherente con dicho objetivo.

En los años siguientes las finanzas públicas se mantuvieron en una situación de relativo equilibrio (véase Cuadro $\mathrm{N}^{\circ} 2$ ), mientras que la inflación cayó sostenidamente, aunque en 1980 - tras un año y medio de fijación cambiaria - estaba en torno a 30\%. En buena medida esta rebeldía de la inflación se explica por el aumento experimentado por una inflación mundial en dólares, que reflejaba el efecto de la mayor inflación en los Estados Unidos y el deterioro del dólar en los mercados internacionales como consecuencia de ello. Este proceso sólo se detuvo tras la aplicación del severo ajuste monetario impulsado por Volcker.

Durante los 80, la trayectoria de la inflación se vio directamente afectada por el quiebre en la regla de tipo de cambio nominal fijo que tuvo lugar a mediados de 1982, como consecuencia de la fuerte apreciación experimentada por el dólar en los mercados internacionales, proceso que estaba originando un severo ajuste en la actividad y el empleo. La mezcla del quiebre en la regla cambiaria con el proceso recesivo que estaba en marcha, produjo

${ }^{41}$ A partir de 1976 la tasa de crecimiento del dinero (M1) cae sostenidamente hasta el año 1980, cuando ésta alcanza a 64,05\%, luego de crecer en 57,8\% el año previo. Una explicación razonable de ello es la monetización del fuerte influjo de capitales que se produjo tras la fijación del tipo de cambio nominal, a mediados de 1979. La fuente de las cifras monetarias es Díaz, Lüders y Wagner (2002).

${ }^{42}$ Para algunos economistas, las fragilidades del mercado financiero — desde los orígenes del proceso de liberalización — se explican por la debilidad que mostraban las empresas que habían sido tomadas o estatizadas durante el gobierno de la Unidad Popular, las que trasladaron al mercado necesidades de financiamiento que anteriormente cubría el gobierno a través de su déficit. Otros han destacado la presencia de problemas del tipo moral hazard como resultado de la misma acción de las autoridades, frente a los primeros indicios de dificultades en el sistema. Al respecto véase, por ejemplo, Harberger (1984). 
CUADRO N 2

\begin{tabular}{|c|c|c|c|c|}
\hline & $\begin{array}{c}\text { Inflación } \\
\mathrm{L}^{1}\end{array}$ & $\begin{array}{l}\text { Superávit } \\
\text { SPNF L }{ }^{2}\end{array}$ & $\begin{array}{l}\text { Crecimiento } \\
\text { PIB L }{ }^{3}\end{array}$ & $\begin{array}{c}\text { Tipo de } \\
\text { cambio real } \mathrm{L}^{4}\end{array}$ \\
\hline 1970 & 34,9 & $-6,7$ & $2,1 *$ & n.d. \\
\hline 1971 & 22,1 & $-15,3$ & $9,0 *$ & n.d. \\
\hline 1972 & 163,4 & $-24,5$ & $-1,2 *$ & n.d. \\
\hline 1973 & 508,1 & $-30,5$ & $-5,6^{*}$ & n.d. \\
\hline 1974 & 375,9 & $-5,4$ & $1 *$ & n.d. \\
\hline 1975 & 340,7 & $-2,0$ & $-13,3$ & n.d. \\
\hline 1976 & 174,3 & 4,0 & 3,2 & n.d. \\
\hline 1977 & 63,5 & 0,4 & 8,3 & 57,1 \\
\hline 1978 & 30,3 & 1,6 & 7,8 & 68,1 \\
\hline 1979 & 38,9 & 4,8 & 7,1 & 70,2 \\
\hline 1980 & 31,2 & 6,1 & 7,7 & 60,8 \\
\hline 1981 & 9,5 & 0,8 & 6,7 & 52,9 \\
\hline 1982 & 20,7 & $-3,4$ & $-13,4$ & 59,0 \\
\hline 1983 & 23,1 & $-3,0$ & $-3,5$ & 70,8 \\
\hline 1984 & 23,0 & $-4,3$ & 6,1 & 74,0 \\
\hline 1985 & 26,4 & $-2,6$ & 3,5 & 90,9 \\
\hline 1986 & 17,4 & $-2,1$ & 5,6 & 100,0 \\
\hline 1987 & 21,5 & $-0,2$ & 6,6 & 104,3 \\
\hline 1988 & 12,7 & 0,2 & 7,3 & 111,2 \\
\hline 1989 & 21,4 & 1,3 & 10,6 & 108,6 \\
\hline 1990 & 27,3 & 3,6 & 3,7 & 112,7 \\
\hline
\end{tabular}

$\mathrm{L}^{1}$ Variación diciembre a diciembre. Fuente: INE.

$\mathrm{L}^{2}$ SPNF = Sector público no financiero. Fuente: Larraín y Vergara (2000).

$\mathrm{L}^{3} *$ Fuente: Díaz, Lüders y Wagner (2002) y Banco Central.

$\mathrm{L}^{4}$ Fuente: Banco Central.

una importante crisis financiera ${ }^{43}$, la que dificultó la gestión de la política monetaria en los años siguientes. Sólo a partir de los 90 — especialmente en la segunda parte de dicha década - se observa un acelerado proceso de caída en la inflación, el que coincide con la trayectoria que observó esta variable a nivel mundial, como se puede apreciar en el Cuadro $\mathrm{N}^{\circ} 1$-A.

Más que analizar la trayectoria seguida por la inflación y sus determinantes, nuestro propósito es examinar los factores que la impulsaron en Chile, de modo de establecer qué tan definitivos son los progresos alcanzados en este ámbito. Como consideración general, se postula que la inflación sólo se redujo en forma significativa una vez que se crearon las condiciones para que la política monetaria pudiera enfocarse a este objetivo y no a otro. En efecto, al revisar la experiencia macroeconómica chilena de las últimas décadas se descubre que en una etapa de ésta la política monetaria se subordinó — esencialmente— al financiamiento del gasto público, situación

\footnotetext{
${ }^{43}$ Al respecto véanse Sanhueza (1999) y Ramírez y Rosende (1992).
} 
que es particularmente nítida a comienzos de los 70. Luego, la adopción de diferentes estrategias de fijación cambiaria — “tablita”, tipo de cambio fijo, regla de tipo de cambio real y bandas cambiarias - redujo, en diferentes grados, la posibilidad de hacer uso de la política monetaria para contener la inflación.

En el caso de la fijación del tipo de cambio nominal, es claro que dicha estrategia permitía converger a la inflación mundial relevante, definiéndose con esta política un "ancla” nominal para la economía. No obstante, el complejo escenario financiero internacional de fines de los 70 y comienzos de los 80, donde el ajuste monetario implementado en los Estados Unidos fue un aspecto central, impidió sostener dicha regla. Más tarde, la adopción de una "política de tipo de cambio real”44 dejó la política monetaria "amarrada" a las consecuencias de fijar un precio relativo y, por lo tanto, la economía quedó sin una "ancla nominal".

Si bien tras la crisis de comienzos de los 80 se estructuró un programa macroeconómico coherente, donde el establecimiento de una regla de tipo de cambio real era apoyada por una política fiscal austera, en el contexto de una activa agenda de reformas macroeconómicas dirigidas a elevar la productividad de la economía, la armonización de la regla cambiaria con una trayectoria declinante de la inflación se hizo progresivamente más difícil en la medida en que la recuperación de la economía atrajo capitales que empujaron al alza el gasto y la inflación. Así, sólo hacia fines de los 90, al adoptarse un esquema de flotación cambiaria, en un marco donde el Banco Central estaba legalmente inhabilitado para hacer préstamos al fisco, se configuró un escenario coherente con el logro de una tasa de inflación similar a la existente en las economías industrializadas. Esto ocurrió en un contexto de presiones deflacionarias a nivel mundial —originadas en una expansión del comercio mundial, como se indicó antes-, lo que evidentemente contribuyó a los progresos alcanzados.

Este cambio en el marco dentro del cual se inserta la política monetaria hizo posible reducir — en forma significativa — la inercia inflacionaria existente, la que dificultaba el éxito de los programas de estabilización. Ello al menos en términos de la velocidad con la que se manifestaban sus resultados. Este punto es destacado por Edwards (2000), quien plantea que la existencia de un mecanismo de indexación del tipo de cambio, durante buena parte del programa de estabilización iniciado a mediados de los 70, conspiró contra el logro de mayores progresos en el control de la inflación.

${ }^{44}$ Ello con el propósito de revitalizar el sector transable de la economía, el que se había visto seriamente afectado por la fuerte caída que experimentó el tipo de cambio real en 1981, como consecuencia de un fuerte influjo de capitales. 
Si bien parece necesario un análisis más acabado para establecer las consecuencias de los mecanismos de ajuste del tipo de cambio nominal entonces vigentes — en la medida en que un juicio más preciso requiere de alguna idea respecto a cuál fue la magnitud de los desequilibrios que dicha fórmula podría haber provocado entre el tipo de cambio real efectivo y el de "equilibrio"-, parece en general correcto sostener que este factor dificultó el proceso de control de la inflación en Chile.

La prolongada historia inflacionaria de la economía chilena, que llevó a un extendido uso de mecanismos de indexación, hace difícil suponer que la aplicación de una política monetaria expansiva —en el contexto de tasas de inflación anual de 15\% o más - pueda ser explicada por el intento de explotar una curva de Phillips, en la línea del modelo de Kydland y Prescott antes analizado. Ello por cuanto en el contexto mencionado parece razonable suponer que el parámetro $\theta$ de la ecuación (6) era particularmente bajo. Luego, el análisis de la trayectoria seguida por la inflación en esta economía hace necesario examinar otros aspectos detrás de este proceso, tal como la estructura institucional dentro de la cual se insertó el manejo monetario, lo que se describe —en términos generales— a continuación.

\subsection{Dinero pasivo I: Teoría fiscal de la inflación}

Como se indicó, una de las causas importantes detrás del elevado crecimiento monetario que empujaba la inflación en Chile, fue la presencia de un persistente déficit del sector público, el que presionaba por los recursos provenientes de la creación de dinero. La relación entre el cuadro de finanzas públicas y la inflación se puede expresar de un modo sencillo en la ecuación (10), que presenta la restricción presupuestaria del gobierno, medida en términos reales:

$$
\mathrm{G}=\mathrm{tZ}+\varphi \mathrm{qX}+\mathrm{dM} / \mathrm{P}+\mathrm{dB} / \mathrm{P} .
$$

El primer término indica los ingresos corrientes —no cobre- del gobierno, donde " $\mathrm{t}$ " representa un vector de tasas de impuestos y "Z" la base de estos. De este modo, un manejo eficiente de las finanzas públicas requeriría encontrar aquel conjunto de impuestos — tasas y bases- que minimizando las distorsiones que éstos ocasionan en la asignación de recursos, maximicen la recaudación. A comienzos de los 70 éste era un problema importante en la gestión de las finanzas públicas, puesto que la estructura de impuestos — bases y el nivel de las tasas establecidas - eran 
incompatibles con criterios de eficiencia ${ }^{45}$. En este contexto, era habitual la verificación de déficit en las cuentas fiscales, el que hacía necesario acudir al financiamiento monetario, corroborándose —en alguna medida— la hipótesis planteada por Cukierman, Edwards y Tabellini (1992), en cuanto a que en diversas economías, especialmente en desarrollo, la inflación se originó en conflictos y polarización política, que impidieron establecer una esquema tributario más eficiente para financiar el presupuesto fiscal ${ }^{46}$.

El segundo término de la ecuación (12) es especialmente relevante para el análisis de las finanzas públicas de Chile, e indica los ingresos provenientes del cobre, donde " $\varphi$ " es la tasa de impuesto sobre las exportaciones de cobre, el que tras la estatización de la gran minería del cobre -a comienzos de los 70 - pasó a ser cercano a 1, reflejando una tasa de $100 \%$; “q" expresa el precio internacional de ésta materia prima y " $\mathrm{X}$ ” la cantidad exportada.

El tercer término es la recaudación que obtiene el gobierno por concepto de creación de dinero, la que también se puede escribir como

$$
\mathrm{dM} / \mathrm{P}=(\mathrm{dM} / \mathrm{M})(\mathrm{M} / \mathrm{P})=\left(\pi+\eta_{\mathrm{y}}\right) \mathrm{m}^{47}
$$

donde $\mathrm{M} / \mathrm{P}=\mathrm{m}$

gy = tasa de crecimiento del producto real

$\eta=$ elasticidad ingreso de la demanda de dinero.

Por simplicidad, se supondrá que las posibilidades de endeudamiento — externo y/o interno- del gobierno son exógenas e iguales a $\Phi$.

Despejando entonces para la inflación se tiene:

$$
=(1 / m)[G-t Z-\varphi q X-\operatorname{mgym}-\Phi] .
$$

De acuerdo con (14), la presión sobre la política monetaria — y consecuentemente sobre la inflación ${ }^{48}$ — se intensificaría en aquellos períodos en que los términos de intercambio sufrieran un deterioro importante, o

\footnotetext{
${ }^{45} \mathrm{Al}$ respecto véase Larraín y Vergara (2000).

${ }^{46}$ De acuerdo con esta hipótesis, el clima de conflicto político impedía materializar acuerdos conducentes a revisar el sistema tributario y/o la eficiencia del gasto fiscal, lo que hacía inevitable la monetización de los déficit del sector público.

${ }^{47}$ En equilibrio $\mathrm{dM} / \mathrm{M}=\mu=(\pi+\eta \mathrm{g} y)$.

${ }^{48}$ En una economía marcada por una larga historia inflacionaria, la velocidad a la cual los incrementos en la tasa de expansión del dinero se traducían en mayor ritmo de crecimiento de los precios era relativamente rápida. Por la misma razón, lo contrario ocurría para desaceleraciones en la tasa de crecimiento del dinero.
} 
cuando lo hiciera la tasa de crecimiento de la economía. Este enfoque fiscal de la inflación, donde el gobierno utiliza la política monetaria para financiar gasto o acumular reservas internacionales, no sólo parece coherente con la historia de la economía chilena - $\mathrm{O}$ al menos en una parte importante de ésta- sino que además permite explicar el comportamiento anticíclico de la inflación que, como se mencionó anteriormente, encuentran algunos estudios, lo que deja de manifiesto una importante sustitución entre el financiamiento monetario ${ }^{49}$ y los recursos generados por las ventas de cobre al exterior.

Al examinar la trayectoria de la inflación desde la perspectiva de la ecuación (14), se concluye que sólo es posible lograr una caída duradera en ésta en la medida en que se produzca un cambio estructural en el diseño de las finanzas públicas ${ }^{50}$, de modo de conseguir un crecimiento más armónico entre los gastos y los ingresos corrientes $(\mathrm{tZ}+\varphi \mathrm{qX})^{51}$. En el caso chileno ello ocurrió a partir de mediados de los 70 , como se puede apreciar en el Cuadro $\mathrm{N}^{\circ} 2$, no obstante lo cual la inflación se mantuvo en tasas elevadas - de dos dígitos- por un período prolongado, lo que hace necesario pensar en interpretaciones complementarias a ésta.

\subsection{Dinero pasivo II: Indeterminación nominal}

Como se indicó, tras un severo proceso de ajuste y ordenamiento, a mitad de los 70 la economía chilena había logrado progresos importantes en el proceso de ordenamiento de las finanzas públicas ${ }^{52}$. Esto hizo posible la fijación del tipo de cambio nominal en junio de 1979, lo que se esperaba ayudaría a contener la dinámica inflacionaria existente en los arraigados esquemas de indexación de precios y salarios. Por otro lado, la fijación del tipo de cambio permitiría reducir las muy elevadas tasas reales de interés prevalecientes, en el supuesto de que este fenómeno se explicaba —en buena medida - por expectativas de un repunte de la inflación.

Luego de un período inicial de caída en la inflación, el escenario observó un cambio importante tras el ajuste monetario en los EE.UU. antes

\footnotetext{
${ }^{49}$ Por ejemplo, véase Rosende y Guier (1994).

${ }^{50}$ Este punto es destacado por Sargent (1986) en su evaluación de los programas de estabilización de Margaret Thatcher en Inglaterra a mediados de los 80 y por el Primer Ministro de Francia, Raymond Poincaré, a mediados de los 20.

51 Se omite de la expresión de los "ingresos corrientes" la recaudación por concepto de señoreaje social, aunque en un esquema de banco central "dependiente" ésta debe incluirse.

52 Véase Larraín y Vergara (2000).
} 
comentado, el que — como se indicó- dio origen a una fuerte apreciación del dólar en los mercados internacionales, en un contexto recesivo internacional. En el plano doméstico, este cambio en la política monetaria de los EE.UU. provocó una aguda crisis cambiaria y financiera, la que a su vez ocasionó un importante déficit cuasifiscal ${ }^{53}$, en un contexto donde la inflación repuntaba. Ello como resultado de sucesivas devaluaciones del peso que tuvieron lugar tras el quiebre de la regla cambiaria en junio de 1982.

La crisis financiera, que llevó a intervenir una serie de bancos a comienzos del año 1983, parece haber provocado un importante grado de duda en los agentes económicos respecto a las posibilidades de que el Banco Central pudiera conservar el control de la política monetaria - y por lo tanto de la inflación - en este contexto. Ello a juzgar por la persistencia de la serie misma de inflación, como resultado de un extendido uso de los mecanismos de indexación en los diferentes tipos de contratos de la economía.

En los meses siguientes a la irrupción de la crisis cambiara-financiera, el Gobierno de Chile acordó un programa de ajuste con el Fondo Monetario Internacional, el que incluía como un aspecto importante la mantención de un tipo de cambio "competitivo" y un manejo monetario coherente con una caída gradual de la inflación. Si el bien el tema inflacionario no fue el eje central de dicho programa, sino que más bien la solución de los problemas de balanza de pagos que planteaba un alto endeudamiento externo, el programa de ajuste se proponía ir avanzando en el logro de un crecimiento más lento de los precios, aunque el control de la política monetaria ${ }^{54}$ quedó en alguna medida limitado por la adopción de un sistema de fijación cambiaria conducente a lograr cierta estabilidad del tipo de cambio real.

A medida que la economía avanzó en su proceso de recuperación, la mencionada regla cambiaria se hizo cada vez más incompatible con el control de la política monetaria y con ello de la inflación ${ }^{55}$, en la medida en que el mejoramiento de las condiciones macroeconómicas impulsaba un mayor gasto privado, lo que hacía necesaria una caída del tipo de cambio real. Sin

\footnotetext{
${ }^{53}$ Ello debido a que dentro del proceso de saneamiento de la banca y normalización del crédito, el Banco Central compró una fracción importante de la "cartera mala" de los bancos, la que intercambió por deuda propia, que se fijó en términos reales y prometía una tasa de interés predeterminada. Respecto a este proceso, véase Reinstein y Rosende (2000).

${ }^{54}$ Cabe recordar que, en el caso argentino, la crisis financiera generó una fuerte inflación, lo que se interpretó como el propósito de las autoridades por reducir el valor real de las deudas - “licuación” de éstas - recorriendo el camino inverso del fenómeno de deuda-deflación reportado por Irving Fisher en los años 30.

${ }^{55}$ Sobre este conflicto, véase Rosende (1985).
} 
embargo, la regla cambiaria impedía que ello ocurriera, dando origen a presiones inflacionarias, como consecuencia de la expansión de la oferta monetaria que provocaba un tipo de cambio real por encima de su valor de equilibrio ${ }^{56}$.

Este escenario macroeconómico llevó a un extendido uso de modelos de inflación ${ }^{57}$ del tipo "escandinavo-australiano", en la medida en que se mezclaba la lógica de costos del primero, con el tradicional modelo de determinación del tipo de cambio real de Salter-Swan, conocido como "modelo australiano".

Del "modelo australiano" antes mencionado, se llega a ecuaciones del tipo

(15) $\quad \pi_{\mathrm{n}}(\mathrm{t})=\alpha_{0} \lambda(\mathrm{t})+\alpha_{1} \pi^{*}(\mathrm{t})+\gamma \mathrm{gX}(\mathrm{t})+\varepsilon(\mathrm{t})$

las que en términos generales —introduciendo rezagos— se plantean como:

$$
\pi(t)=\alpha 0 \lambda(t)+\alpha 1 \pi^{*}(t)+\varphi Z(t)+\rho \pi(t-1)+v(t)
$$

donde:

$\pi_{\mathrm{n}}=$ tasa de inflación de los bienes no transables.

$\lambda=$ tasa de devaluación del tipo de cambio nominal.

$\pi^{*}=$ tasa de inflación internacional de los bienes transables ${ }^{58}$.

gX = tasa de variación en la razón Gasto Agregado a PIB.

$\varepsilon=$ término aleatorio.

$\pi=$ tasa general de inflación.

$\mathrm{Z}=$ indicador de presiones de demanda ${ }^{59}$.

A pesar de la evidente utilidad de este tipo de modelos para evaluar el comportamiento de la inflación en el corto plazo, una limitación importan-

${ }^{56}$ El problema se hacía especialmente agudo cuando se vislumbraba la posibilidad de alguna corrección en la política cambiaria para acomodar un tipo de cambio real más bajo. Cabe recordar que en presencia de bienes no transables se tiene que: $r(t)=r^{*}(t)+$ $\sigma$ de $(\mathrm{t}+1)$, donde: $\mathrm{r}=$ tasa real de interés doméstica; tasa real de interés externa; $\mathrm{e}=$ tipo de cambio real; $\sigma=$ participación de los bienes no transables en la canasta de consumo del agente representativo.

${ }^{57} \mathrm{Al}$ respecto véanse los artículos que aparecen en el volumen editado por Morandé y Rosende (1995).

${ }^{58}$ Habitualmente se utiliza algún indicador de inflación externa relevante.

${ }^{59}$ En algunos estudios se utiliza un indicador de exceso de liquidez, por ejemplo en Edwards (2000), mientras que en otros, como Corbo y Piedrabuena (1995), se usan indicadores de exceso de demanda en la línea de la "brecha de producto". 
te de los mismos es que, en general, omiten una discusión más profunda respecto a los determinantes últimos de ésta. En particular, acerca de la forma en que los arreglos institucionales condicionan el grado de control que tiene el banco central de la política monetaria y con ello de la inflación. En otras palabras, un análisis del tipo "contabilidad de la inflación” como los indicados en (15'), no permite establecer la forma en que la estructura de incentivos y restricciones dentro de la cual se desenvuelven las autoridades monetarias influye sobre la trayectoria de la inflación en el mediano plazo. Así, por ejemplo, en un contexto donde es restrictiva una cierta regla cambiaria $^{60}$, un shock exógeno que lleve a la autoridad monetaria a expandir la liquidez provocará movimientos en la inflación que no responden a una estrategia explícita de política monetaria ${ }^{61}$, del tipo examinado en la sección 3. En realidad en este caso no hay política monetaria en un sentido estricto y el dinero es pasivo.

Por otra parte, los agentes racionales descubrirán la relativa precariedad de los progresos en el control de la inflación, en la medida en que shocks transitorios de costos ocasionen ciclos en ésta, los que pueden llegar a ser prolongados, dado la regla cambiaria vigente y el extendido uso de mecanismos de indexación en los contratos.

$\mathrm{Al}$ revisar la gestión de la política monetaria en los 90 se comprueban las dificultades que planteó la existencia de una regla de tipo de cambio real para ejercicio de un manejo monetario independiente ${ }^{62}$. Una manifestación clara de este conflicto son los frecuentes ajustes de la regla cambiaria; una masiva acumulación de reservas internacionales y la emisión de deuda interna para atenuar el efecto monetario del proceso. Cabe mencionar que como una forma de conciliar la regla cambiaria existente con el ejercicio de la política monetaria, el Banco Central estableció a comienzos de los 90 un encaje $^{63}$ —no remunerado— a los créditos externos.

${ }^{60}$ Por ejemplo, en un esquema de bandas cuando el tipo de cambio se "pega" al límite inferior de ésta, como ocurrió a menudo en Chile en la segunda mitad de los 90.

${ }^{61}$ En el contexto de un modelo del tipo VAR, se obtendrá que la inflación responde a factores diferentes a la política monetaria, como podrían ser cambios en los precios internacionales de algunos productos o en la absorción fiscal. Esta última podría influir sobre la trayectoria de la política monetaria y la inflación, aun en presencia de un presupuesto fiscal equilibrado, en la medida que influya sobre el tipo de cambio real de equilibrio en un esquema de fijación de esta variable, por ejemplo.

${ }^{62} \mathrm{Al}$ respecto véanse Rosende (1998) y Fontaine (2000).

${ }^{63}$ Este encaje fue introducido en 1991 con una tasa de $20 \%$ y se aplicó sólo a los créditos externos. En 1992 se elevó a 30\% y se extendió a los depósitos en moneda extranjera. En 1994 se aplicó a las compras de acciones vía ADR y en 1995 a toda la inversión extranjera considerada "financiera”. En 1998 el encaje se eliminó. 


\section{El esquema de metas inflacionarias}

Durante los últimos años ha adquirido cierta popularidad entre los bancos centrales la idea de establecer metas para la inflación, como elemento orientador de la política monetaria ${ }^{64}$. De acuerdo con este esquema, se define un cierto objetivo de inflación hacia el cual debe apuntar dicha política, reconociendo la existencia de rezagos en la manifestación de sus efectos y, por otro lado, dejando abierta la posibilidad de acomodar shocks que pudieran afectar en el corto plazo el comportamiento de la actividad y el empleo. Se trata entonces de un marco de referencia que incorpora los planteamientos "neoclásicos", en cuanto a que la política monetaria no debe apuntar al logro de objetivos reales, por cuanto su influencia en éstos es transitoria y los costos del intento por influirlos pueden ser significativos. En esta perspectiva, lo razonable es perseguir ciertos objetivos en materia de inflación, especialmente si se estima que la demanda por dinero es inestable, lo que haría desaconsejable utilizar alguna regla de crecimiento para algún agregado monetario.

Por otro lado, este esquema deja el espacio para hacer uso de la política monetaria con el propósito de atenuar el efecto de cambios imprevistos en las condiciones reales de la economía, considerando que la capacidad de respuesta de los precios a éste sería imperfecta, en línea con la tradición keynesiana.

Un aspecto esencial dentro del análisis del funcionamiento de este tipo de estrategia, se refiere a la naturaleza de los costos que debe enfrentar un banco central que no cumple con los objetivos inflacionarios planteados para un cierto período. Si éstos son elevados, como se planteó por ejemplo en el caso del esquema de contratos neozelandés ${ }^{65}$, que contemplaba costos en dinero y el eventual despido del presidente del banco central, la figura es muy diferente al caso en que este incumplimiento no tiene consecuencias concretas, más allá de un mal rato en el Parlamento para la autoridad monetaria. Éste es un aspecto importante de considerar en la evaluación de este esquema, puesto que es habitual que se incluyan dentro de la categoría de inflation targeters a economías donde el compromiso de sus bancos centrales con dicho objetivo es muy diferente, no obstante coincidan en su preocupación por el mismo y en la materialización de progresos en este ámbito.

${ }^{64} \mathrm{Al}$ respecto véanse los trabajos publicados en el volumen editado por Loayza y Soto (2002).

${ }^{65} \mathrm{Al}$ respecto véanse Drew (2002) y Walsh (1995). 
La experiencia chilena

La ley que estableció la autonomía del Banco Central de Chile, en vigencia desde 1990, plantea como objetivo de esta institución "cautelar por la estabilidad en el valor de la moneda y por la estabilidad de los pagos internos y externos", lo que puede interpretarse como un mandato claro en orden a promover ciertos objetivos en materia de inflación, de modo de avanzar hacia un cuadro de estabilidad de precios. Sin embargo, no parece razonable inferir de aquí que a partir de dicho año se adoptó un esquema de metas de inflación. Como se indicó antes, en los primeros años de funcionamiento de dicho cuerpo legal se produjo un cierto grado de controversia respecto a la forma en que debía interpretarse el mencionado mandato, en especial, en lo que se refiere a “... la estabilidad de los pagos internos y externos”. De hecho, en esta etapa la autoridad monetaria llevó a cabo una activa intervención en el mercado cambiario, con el propósito de cumplir con un cierto objetivo de tipo de cambio real, como se indicó antes.

Tanto Morandé (2002), como Corbo, Landerretche y Schmidt-Hebbel (2002), destacan el aporte del establecimiento de un esquema de metas de inflación en la caída que experimentó esta variable en Chile desde comienzos de los 90. Este esquema habría permitido "anclar" las expectativas inflacionarias del público al tiempo que dejaba espacio para acomodar shocks reales, de modo de minimizar su efecto adverso sobre la actividad.

Para Morandé (2002), la puesta en marcha de la ley que establecía la autonomía del Banco Central, promovió un movimiento gradual hacia el establecimiento de un esquema de "metas de inflación". Identifica una primera etapa dentro del proceso de configuración de este esquema - entre septiembre de 1990 y septiembre de 1999- período en el cual la autoridad monetaria fue asumiendo anualmente compromisos en materia de inflación. Como se indicó, en dicho período el Banco Central debió hacer frente a la restricción que planteaba la existencia de un tipo de cambio fijado. La segunda etapa se inicia — de acuerdo a Morandé- en septiembre de 1999, cuando se deja libre el tipo de cambio y la gestión de la política monetaria se orienta exclusivamente al cumplimiento de la meta inflacionaria.

Aun cuando es razonable suponer que el establecimiento de un nuevo marco institucional para el Banco Central, el que impedía la repetición de episodios de financiamiento monetario de las finanzas públicas, puede haber contribuido a la reducción de las expectativas inflacionarias a partir de 1990, no parece serlo el suponer que la entrega de "proyecciones-compromiso" por parte de la autoridad monetaria al Senado representaba en sí un cambio en el régimen de política monetaria. Por otro lado, el análisis de los factores que explican la reducción que experimentó el ritmo de crecimiento 
de los precios en los 90, necesariamente debe contrastar los argumentos que destacan el efecto del cambio institucional antes mencionado, con una fuerte y sostenida presión a la baja en el tipo de cambio nominal, la que contribuyó en forma significativa a la caída de la inflación, sin que ello ocasionara costos de actividad importantes. Esta hipótesis es destacada por Calvo y Mendoza (1999) y Rosende (1998).

Las causas para la presión a la baja del tipo de cambio son diversas, pero en lo esencial se resumen en la confianza que generó en los inversionistas el compromiso de las autoridades económicas entrantes en 1990, en orden a mantener una economía abierta al exterior, en la que el sector privado sería el actor principal. Adicionalmente, es importante señalar que a fines del año 1989 el tipo de cambio real ya mostraba una tendencia a la apreciación real del peso, la que al estar limitada por la regla cambiaria vigente generaba una cierta presión inflacionaria ${ }^{66}$, la que desaparecería una vez que dicha regla fuese corregida, como ocurrió más tarde.

Pese a la importancia de los cambios introducidos en el marco de la política monetaria a comienzos de los 90, hacia mitad de la década no se detectaba un cambio estructural en la trayectoria de la inflación ${ }^{67}$, de acuerdo con la evidencia que entregan los estudios econométricos publicados en el volumen editado por Morandé y Rosende (1995). Más aun, en algunos de los trabajos se discute acerca de si la caída que esta variable mostraba en la primera parte de la década era "permanente" o "transitoria", sin que las conclusiones fuesen claras. Por otro lado, ninguno de los ocho estudios publicados en dicho volumen hace referencia a eventuales "metas de inflación" de la autoridad monetaria, por lo que si bien es razonable reconocer el valor y peso de las proyecciones que cada año —en el mes de septiembreentregaba el Banco Central al Senado, éstas habrían adquirido el carácter de tal tras el severo ajuste de fines de década, donde quedó claramente de manifiesto el compromiso de la política monetaria con dicho objetivo.

Para ilustrar la importancia que parecen haber tenido los shocks reales de oferta en el proceso de caída de la inflación registrado en Chile en los 90, cabe señalar que de acuerdo con las simulaciones que realizan SchmidtHebbel y Servén (1995) —en el contexto de un modelo de equilibrio general

${ }^{66}$ Por otro lado, era difícil que se pudiera sostener el programa de conversión de deuda que se había iniciado en los años previos, generando una importante demanda por dólares y con ello ayudando a la mantención de un tipo de cambio real elevado. Al respecto véase Rosende (1993).

${ }^{67}$ Cabe señalar que las nuevas autoridades monetarias aplicaron un severo ajuste a pocas semanas de asumir la conducción de este organismo, con el propósito de contener el aumento que registraba la inflación en los trimestres previos, lo que parece haber ayudado a la configuración de un clima de mayor credibilidad tanto en el esquema institucional como en las nuevas autoridades. 
con expectativas racionales-, una caída de la inflación desde una tasa anual de $14 \%$ a un 5\% ocasionaría importantes costos reales en la medida en que se quisiera materializar ésta en un período breve, a través de una política monetaria contractiva. Considerando que la inflación se redujo desde una tasa de 12,2\% en 1993 a un 6,6\% en 1996, en un contexto de fuerte crecimiento de la actividad y de un manejo monetario expansivo, se confirma la hipótesis de que dicho proceso se vio apoyado por la presencia de shocks favorables de oferta y/o el reacomodo del tipo de cambio nominal a un nivel más coherente con lo que parecía ser el tipo de cambio real de equilibrio.

El trabajo de Rojas, Rosende y Vergara (1995), muestra que hasta mediados de los 90 — período en que termina la muestra considerada en el estudio - la inflación continúa mostrando un grado importante de inercia ${ }^{68}$, en tanto que la trayectoria inflacionaria sólo muestra una tendencia decreciente a partir de mediados de 1993, la que se mantiene hasta junio de 1994, último mes considerado en el estudio. De acuerdo con los autores, esta etapa coincide con una abundancia de divisas y presión a la baja del tipo de cambio.

En Calvo y Mendoza (1999) se cuestiona la hipótesis de que la caída de la inflación observada durante los 90 hubiese sido consecuencia de la aplicación de una política monetaria coherente con dicho objetivo, a través de los ajustes necesarios en la tasa de política monetaria. Sin embargo, destacan la contribución a dicho resultado del proceso de ajuste a la baja del tipo de cambio real en dicho período:

Al igual que les sucedió antes a otros autores, no logramos identificar una conexión sistemática entre un ajuste monetario vía aumentos en las tasas de interés en UF y la desaceleración observada en la inflación, aun cuando el sector de la economía real sí responde a cambios en las tasas en UF. Sin embargo, si se considera que el marco de la política monetaria está siendo influenciado por la evolución de la tasa de cambio, se encuentra evidencia de una conexión dinámica, sistemática, entre la apreciación nominal y real del tipo de cambio y la caída de la inflación ${ }^{69}$.

${ }^{68}$ En este estudio se usa un indicador de “inflación subyacente” como eje del análisis.

69 "Like other authors before us, we failed to identify a systematic connection between monetary tightening via increases in UF interest rates and the observed deceleration of inflation, even though the real economy does respond to changes in UF rates. If the monetary policy framework is interpreted instead as being influenced by the evolution of the exchange rate, there is some evidence of a systematic, dynamic connection between the appreciation of real and nominal exchange rates and the fall in inflation", Calvo y Mendoza (1999), p. 34. 
Un aspecto importante de considerar dentro del análisis de la experiencia de control de la inflación en Chile, se refiere al episodio de severa contracción monetaria que tuvo lugar a fines de los 90. Éste se originó en un cambio adverso en la coyuntura internacional ${ }^{70}$, el que encontró a la economía chilena con un importante desequilibrio en las finanzas públicas como resultado de un manejo fiscal marcadamente expansivo. La combinación de ambos elementos provocó un deterioro importante de la cuenta corriente de la balanza de pagos, junto con una fuerte presión al alza del tipo de cambio. Con el propósito de evitar que el "ataque" contra el peso se transformara en un elemento desestabilizador de las expectativas inflacionarias que llevase a retroceder en los avances logrados en esta materia, el Banco Central aplicó un severo ajuste monetario ${ }^{71}$, lo que se tradujo en un significativo exceso de demanda de dinero, el que ocasionó una fuerte desaceleración de la actividad en el año 1998 y una leve recesión el año 1999.

Más allá de la discusión acerca de la forma en que se condujo la política monetaria en el episodio mencionado, parece razonable suponer que éste representó un antecedente importante dentro del proceso de formación de expectativas del público, por cuanto probó al mercado la solidez del compromiso del Banco Central con el logro de una trayectoria declinante de la inflación. De hecho, a partir de este episodio, que culmina con la liberalización del tipo de cambio, se hace más nítido el objetivo inflacionario en torno al cual se organiza la política monetaria, mientras que las expectativas del mercado - reflejadas en los precios de los instrumentos financierosmuestran una importante credibilidad en los anuncios de la autoridad en esta materia.

\section{La "deflación” importada}

Como se muestra en el Cuadro 1-A, a partir de comienzos de los 90 se observa un extendido proceso de caída de la inflación en el mundo. Si bien éste puede explicarse a partir de algunas de las teorías que ponen el acento en el papel de la política monetaria, en el análisis de dicho resultado no debe quedar fuera la revisión de la influencia de los cambios que ha experimentado la economía mundial en este período. En efecto, durante el mismo se aprecia la influencia de dos potentes cambios en la economía mundial: por un lado, la decidida incorporación al comercio internacional de

\footnotetext{
${ }^{70}$ Este proceso se inicia con la denominada como "crisis asiática”, que se origina en la manifestación de problemas financieros y de cuenta corriente en Tailandia.

${ }^{71} \mathrm{Al}$ respecto, véase Rosende y Tapia (2006).
} 
países como China, India y de aquellos que formaron la antigua Unión Soviética. Por otro lado, se manifiesta con fuerza el efecto de los cambios tecnológicos ocurridos en el ámbito de las tecnologías de información, los que han tenido un importante efecto en el desarrollo del comercio y el manejo de inventarios.

En un reciente informe el Fondo Monetario Internacional ${ }^{72}$ reconoce la presencia de una presión deflacionaria — virtuosa— que se configuró entre mediados de los 90 y la mitad de la presente década, la que sin ser la causa última de la caída de la inflación que muestran muchas economías en el período en cuestión, habría ayudado en este proceso.

En Rosende y Tapia (2006) se analiza la presencia de estos factores externos en el proceso de caída de la inflación experimentada por la economía chilena en la última década. Este análisis adquiere especial interés considerando que durante la primera mitad de la presente década se observó una rápida caída de la inflación en Chile, junto con un fuerte crecimiento de los agregados monetarios y el crédito. Si bien un análisis del aporte relativo de los diferentes factores que influyeron en la caída de la inflación chilena desde los 90 requeriría de un trabajo empírico ad hoc, los antecedentes que muestra el Cuadro $\mathrm{N}^{\circ} 3$ dejan de manifiesto la presencia de un shock deflacionario externo, en el que se mezcla una caída en el precio internacional en dólares de la canasta de bienes de consumo que importa el país y, posteriormente, una caída del tipo de cambio nominal.

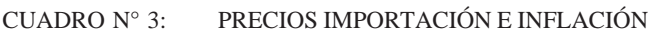

\begin{tabular}{lcccc}
\hline & $\begin{array}{c}\text { Índice de precios } \\
\text { importaciones } \\
\text { bienes de consumo }\end{array}$ & $\begin{array}{c}\text { Tipo de cambio } \\
\text { mominal } \\
\text { promedio }\end{array}$ & $\begin{array}{c}\text { Precio bienes de } \\
\text { consumo importados } \\
\text { en pesos }\end{array}$ & $\begin{array}{c}\text { IPC } \\
\text { promedio }\end{array}$ \\
\hline 1999 & $-5,2$ & 10,5 & 5,3 & 3,3 \\
2000 & $-8,9$ & 6,0 & $-2,9$ & 3,8 \\
2001 & $-5,6$ & 17,7 & 12,1 & 3,6 \\
2002 & $-5,1$ & 8,5 & 3,4 & 2,5 \\
2003 & $-4,4$ & 0,4 & $-4,0$ & 2,8 \\
2004 & 2,0 & $-11,8$ & $-9,8$ & 3,0 \\
2005 & 2,5 & $-8,2$ & $-5,7$ & 3,4 \\
2006 & 1,5 & $-5,3$ & $-3,8$ & 4,4 \\
2007 & 2,2 & $-1,5$ & 0,7 & \\
\hline
\end{tabular}

Fuente: Elaborada a base de datos del Banco Central de Chile.

${ }^{72} \mathrm{Al}$ respecto véase FMI (2005). 
Es importante advertir que las "presiones deflacionarias" que muestra el Cuadro $\mathrm{N}^{\circ} 3$ para la presente década, se producen en un cuadro de amplia credibilidad en el compromiso inflacionario del Banco Central de Chile, dado la fuerza con la que se aplicó el ajuste monetario a fines de la década anterior ${ }^{73}$. La combinación de estos factores puede explicar —al menos en parte- la relativa indiferencia con la que los agentes económicos reaccionaron luego frente a la rápida expansión que registraron los agregados monetarios.

Desde otro punto de vista, la verificación de un fuerte crecimiento de los agregados monetarios en los últimos años, en un contexto donde las expectativas inflacionarias se han mantenido estrechamente cercanas a la meta de inflación del Banco Central hasta comienzos del año 200774, configura un escenario que lleva a suponer que la autoridad ha adoptado -implícitamente- la hipótesis de que dispone de un mayor margen de maniobra para la política monetaria, lo que en términos del sencillo modelo expuesto en la Sección 4 se puede describir como un aumento del parámetro $\theta$ de la ecuación (6). Sin embargo, es importante señalar que esta percepción de un "mayor límite de velocidad" para la política monetaria conlleva el riesgo de que un eventual cambio de las condiciones reales conduzca a un aumento en la inflación ${ }^{75}$ y modifique las expectativas inflacionarias del público. Este escenario podría dar origen a un ciclo inflacionario, en la línea planteada por Sargent, en su interpretación para "la conquista de la inflación" en los Estados Unidos. Ciertamente de aquí surge una interrogante de gran interés académico y de política, la que sólo podrá resolverse a la luz de la propia experiencia y de nuevas investigaciones.

\section{Conclusiones}

Hasta hace algunos años se percibía un clima de confianza y optimismo en la profesión respecto al control inflacionario, el que llevó a algunos economistas a proclamar "el fin de la inflación”, con el mismo entusiasmo con que décadas atrás se proclamó el "fin del ciclo económico". Para algunos, este optimismo se sustentaba en el aprendizaje alcanzado por la profesión en las últimas décadas con respecto a los efectos de la política monetaria, el que al desestimar la existencia de un trade off duradero entre

\footnotetext{
${ }^{73} \mathrm{Al}$ respecto véase Rosende y Tapia (2005).

${ }^{74}$ Este año tiene una serie de particularidades que llevaron a un aumento de la inflación, el que será necesario examinar para establecer sus verdaderas causas y profundidad.

${ }^{75}$ Dado el fuerte crecimiento experimentado por la liquidez y el crédito.
} 
inflación y desempleo, llevó a enfocar esta política al logro de estabilidad de precios, o al menos, en la tasa de crecimiento de éstos.

En el mismo contexto, los progresos alcanzados en la comprensión de los fenómenos monetarios promovió importantes cambios en la institucionalidad dentro de la cual se desenvuelven los bancos centrales - especialmente en términos del otorgamiento de autonomía a éstos respecto del gobierno de turno-- lo que habría permitido consolidar una gestión de la política monetaria apuntada a controlar la inflación y no a otros objetivos respecto a los cuales su efecto es menos relevante y duradero, como el empleo.

El propósito de este trabajo ha sido revisar la investigación que se ha desarrollado en los últimos años con el propósito de explicar el pronunciado ciclo inflacionario que se registró en numerosas economías en los últimos cuarenta años. Más que discutir respecto a las causas últimas de la inflación, donde el tradicional dictum de Milton Friedman en cuanto a que ésta es "siempre y en todas partes un fenómeno monetario" no parece hacer sido desafiado ${ }^{76}$, la discusión se ha enfocado en la estructura de incentivos que rodea el diseño de política monetaria y su efecto en la inflación.

En la literatura reciente subsiste una importante dosis de optimismo acerca de la efectividad de los arreglos institucionales diseñados para mantener controlada la inflación. No obstante, este optimismo aparece más matizado que antes, en tanto se admite - en algunas investigaciones- que la aparición de shocks adversos, que deterioran el desempeño real de la economía, podrían detonar nuevas presiones para la puesta en marcha de un manejo monetario expansivo y el consecuente rebrote inflacionario.

En el caso de la economía chilena, la revisión de la literatura muestra una irrupción — relativamente reciente- de la hipótesis de que la caída experimentada por la inflación a partir de comienzos de los 90 fue causada por el establecimiento de un esquema de "metas de inflación". Sin embargo, el cambio de estrategia de política monetaria — coherente con dicha hipótesis- no fue reconocido por la literatura sino hasta que la verificación de un severo ajuste monetario, a fines de década, provocó un brusco cambio en las expectativas inflacionarias y en la credibilidad del compromiso del Banco Central con dicho objetivo.

Un análisis de la trayectoria seguida por la inflación en el mundo a partir de los 90 deja claramente de manifiesto el carácter global de la caída

${ }^{76}$ Ello pese a que algunos estudios empíricos han encontrado una escasa capacidad predictiva de los movimientos del dinero sobre la inflación en el corto plazo, durante la presente década, lo que ha llevado a enfatizar otros indicadores como la brecha de producto. No obstante ello, parece difícil explicar un fenómeno inflacionario persistente en ausencia de un crecimiento del dinero acorde con éste. 
experimentada por esta variable, proceso en el que han tenido un papel importante las transformaciones ocurridas en el grado de apertura de importantes economías, al igual que el proceso de cambio tecnológico. En esta perspectiva, no parece razonable atribuir la caída experimentada por la inflación en países como Chile en dicho período, exclusivamente a los méritos de la política monetaria, la que ha contado con un importante apoyo externo.

\section{REFERENCIAS}

Acemoglu, D. (2007): "Introduction to Modern Economic Growth". http://econwww.mit.edu/faculty/acemoglu/books.

Barro, R. J. y D. B. Gordon (1983a): “A Positive Theory of Monetary Policy in a NaturalRate Model”. En Journal of Political Economy, 91 (4): 589-610. (1983b): "Rules, Discretion, and Reputation in a Model of Monetary Policy". En Journal of Monetary Economics, 12 (1): 101-121.

Bordo, M. D., C. Erceg, A. Levin, y R. Michaels (2007): “Three Great American Disinflations”. En NBER Working Paper N 12982, March.

Calvo, G. A. y E. G. Mendoza (1999): "Empirical Puzzles of Chilean Stabilization Policy”. En Guillermo Perry y Danny M. Leipziger (eds.), Chile: Recent Policy Lessons and Emerging Challenges. World Bank Institute Development Studies.

Ceccheti, S. G., P. Hooper, B. Kasman, K. L. Schoenholtz y M. W. Watson (2007): Understanding the Evolving Inflation Process. U.S. Monetary Policy Forum 2007, Global Financial Markets. http://research.chicagogsb.edu/igm/docs/2007USMPFReport.pdf.

Clarida, R., J. Galí y M. Gertler (1999): “The Science of Monetary Policy: A New Keynesian Perspective”. En Journal of Economic Perspectives, 37 (4): 16611707.

(2000): "Monetary Policy Rules and Macroeconomic Stability: Evidence and Some Theory”. En The Quarterly Journal of Economics, February: 147-180.

Corbo, V., O. Landerretche y K. Schmidt-Hebbel (2002): "Does Inflation Targeting Make a Difference?”. En N. Loayza y R. Soto (eds.), Inflation Targeting: Design, Performance, Challenges. Banco Central de Chile.

Corbo, V. y B. Piedrabuena (1995): "Reduciendo una Inflación Crónica: Un Modelo de la Inflación Chilena”. En F. Morandé y F. Rosende (eds.), Análisis Empírico de la Inflación en Chile. P. Universdad Católica de Chile-Ilades/Georgetown University.

Cukierman, A. (1992): Central Bank Strategy, Credibility and Independence. The MIT Press.

Cukierman, A., S. Edwards y G. Tabellini (1992): “Seignioriage and Political Instability”. En American Economic Review, June.

Cukierman, A., S. Webb y B. Neyapti (1993): "Measuring the Independence of the Central Bank and its Effects on Policy Outcomes". En The World Bank Economic Review, 6: 353-398.

De Gregorio, J. (2003): “Mucho Dinero y Poca Inflación: Chile y la Evidencia Internacional”. En Cuadernos de Economía, N 121, Año 40: 716-724. 
De Long, B. J. (1996): “America’s Only Peacetime Inflation: The 1970s”. NBER Working Paper $\mathrm{N}^{\circ} \mathrm{H} 0084$, May.

Díaz, J., R. Lüders y G. Wagner (2002): “La República en Cifras: Chile 1810-2000”. Manuscrito, Instituto de Economía P. Universidad Católica de Chile, septiembre.

Drew, A. (2002): "Lessons from Inflation Targeting in New Zealand”. En N. Loayza y R. Soto (eds.), Inflation Targeting: Design, Performance, Challenges. Central Bank of Chile.

Edwards, S. (2000): "Veinticinco Años de Inflación y Estabilización en Chile (19731998)”. En F. Larraín y R. Vergara (eds.), La Transformación Económica de Chile. Santiago: Centro de Estudios Públicos.

Fondo Monetario Internacional (FMI) (2005): "How Has Globalization Affected Inflation?” En World Economic Outlook. FMI. - World Economic Outlook Database 2008. FMI.

Fontaine, J. A. (2000): “Banco Central Autónomo: En Pos de la Estabilidad”. En F. Larraín y R. Vergara (eds.), La Transformación Económica de Chile. Santiago: Centro de Estudios Públicos.

Friedman, M. (1968): “The Role of Monetary Policy”. En American Economic Review, 58 (1), Mar.: 1-17.

(1969): “The Optimun Quantity of Money”. En su The Optimun Quantity of Money and Other Essays. Aldine Publishing Company.

- (1971): “Theoretical Framework for Monetary Analysis”. En Milton Friedman's Monetary Framework: A Debate with his Critics. Robert Gordon (ed.). University of Chicago Press.

(1977): “Nobel Lecture: Inflation and Unemployment”. En Journal of Political Economy, 85(3), June: 451-472.

Fuhrer, J. C. y G. R. Moore (1995): “Inflation Persistence”. En Quarterly Journal of Economics 110 (1), Feb.: 127-159.

García, P. y R. Valdés (2003): “Dinero y Conducción de la Política Monetaria con Metas de Inflación”. En Cuadernos de Economía, № 121, Año 40: 698-706.

Harberger, A. C. (1984): “La Crisis Cambiaria Chilena de 1982”. En Cuadernos de Economía, agosto, $\mathrm{N}^{\circ} 64$.

Ireland, P. N. (1999): "Does the Time-Consistency Problem Explain the Behavior of Inflation in the United States?” En Journal of Monetary Economics, 44 (2), October: 279-291.

Kydland, F. E. y E. C. Prescott (1977): "Rules Rather than Discretion: The Inconsistency of Optimal Plans”. En Journal of Political Economy, 85 (3) June: 473-491. - (1990): "Business Cycles: Real Facts and a Monetary Myth”. En Quarterly Review, Federal Reserve of Minneapolis, Spring.

Larraín, F. y R. Vergara (2000): “Un Cuarto de Siglo de Reformas Fiscales”. En F. Larraín y R. Vergara (eds.), La Transformación Económica de Chile. Santiago: Centro de Estudios Públicos.

Loayza, N. y R. Soto (2002): Inflation Targeting: Design, Performance, Challenges. Central Bank of Chile.

Lucas, Jr. R. E. (1972): “Expectations and the Neutrality of Money”. En Journal of Economic Theory, 4, April: 103-124.

- (1973): "Some International Evidence on Output-Inflation Tradeoffs". En American Economic Review, 63, June: 326-334. 
Lucas, Jr. R. y T. Sargent (eds.) (1981): Rational Expectations and Econometric Practice. The University of Minnesota Press.

Mankiw, N. G. (2001): “U.S. Monetary Policy during the 90s”. Discussion Paper 1927, Harvard Institute of Economic Research, August.

Mishkin, F. S. (2007): “Inflation Dynamics”. Working Paper Series 13147, NBER, June.

Morandé, F. (2002): “A Decade of Inflation Targeting in Chile: Developments, Lessons and Challenges”. En N. Loayza y R. Soto (eds.), Inflation Targeting: Design, Performance, Challenges. Banco Central de Chile.

Morandé, F., C. J. García y C. Johnson (1995): “La Inflación en Chile: Una Nota Estadística”. En F. Morandé y F. Rosende (eds.), Análisis Empírico de la Inflación en Chile. P. Universidad Católica de Chile-Ilades/Georgetown University.

Muth, J. F. (1960): “Optimal Properties of Exponentially Weighted Forecasts”. En Journal of American Statistical Association, Vol. 55: 299-306.

Orphanides, A. (2002): "Monetary Policy Rules and the Great Inflation”. En American Economic Review, № 92, May: 115-120.

Posen, A. (1993): "Why Central Bank Independence Does Not Cause Low Inflation: There Is No Institutional Fix for Politics”. En Richard O’Brien (ed.), Finance and the International Economy, 7: 41-65.

Ramírez, G. y F. Rosende (1992): "Responding to the Collapse: Chilean Banking Legislation after 1983”. En Philip L. Brock (ed.), If Texas Were Chile: A Primer on Banking Reform. A Sequoia Seminar Publication.

Reinstein, A. y F. Rosende (2000): "Reforma Financiera en Chile”. En F. Larraín y R. Vergara (eds.), La Transformación Económica de Chile. Santiago: Centro de Estudios Públicos.

Rojas, P., F. Rosende y R. Vergara (1995): “Dinámica de la Inflación en Chile: Elementos para el Análisis”. En F. Morandé y F. Rosende (eds.), Análisis Empírico de la Inflación en Chile. P. Universdad Católica de Chile-Ilades/Georgetown University.

Romer, Ch. y D. Romer (2002): "The Evolution of Economic Understanding and Postwar Stabilization Policy”. En Rethinking Stabilization Policy. Federal Reserve of Kansas City, Jackson Hole Symposium.

Rosende, F. (1985): “Tipo de Cambio y Salarios Reales: Consideraciones sobre el Caso Chileno”. En Cuadernos de Economía Nº 67, diciembre: 343-355.

(1993): "La Economía Chilena en el Gobierno de la Concertación: Una Evaluación Preliminar”. En Estudios Sociales, № 75, Trimestre 1, Santiago, CPU. - (1998): "Política Monetaria en Chile en los Noventa: Un ejercicio 'No Neutral’”. En Perspectivas en Política, Economía y Gestión, № 1, Vol. 2.

Rosende, F. y J. C. Guier (1994): “Comportamiento de los Precios en el Ciclo Económico”. En Estudios Públicos N 53, verano: 55-82.

Rosende, F. y M. Tapia (2006): "La Caída de la Inflación en Chile: Políticas, Instituciones y Suerte”. Documento de Trabajo $N^{\circ}$ 308, Instituto de Economía. P. Universidad Católica de Chile.

Sanhueza, G. (1999): "La Crisis Financiera de los Años 80 en Chile: Análisis de sus Soluciones y su Costo”. En Economía Chilena, Banco Central de Chile, Vol. 2, $\mathrm{N}^{\circ} 1$, abril.

Sargent, T. J. (1986): “Stopping Moderate Inflations: The Methods of Poincaré and Thatcher”. En su Rational Expectations and Inflation. New York: Harper \& Row Publishers. 
(1999): The Conquest of American Inflation. Princeton University Press.

Sargent, T. J. y U. Söderström (2000): “The Conquest of American Inflation: A Summary”. En Economic Review, 3: 12-45

Schmidt-Hebbel, K. y L. Servén (1995): "Hacia una Menor Inflación en Chile: Contracción Monetaria bajo Expectativas Racionales”. En F. Morandé y F. Rosende (eds.), Análisis Empírico de la Inflación en Chile. P. Universidad Católica de Chile-Ilades/ Georgetown University.

Stock, James H. y M. W. Watson (2003): "Has the Business Cycle Changed? Evidence and Explanations". Federal Reserve Bank of Kansas City Symposium: "Monetary Policy and Uncertainty", Jackson Hole, Wyoming.

Taylor, J. B. (1979): “Staggered Wage Setting in a Macro Model”. En American Economic Review, 69 (2), May: 108-113.

- (1993): "Discretion versus Policy Rules in Practice". En Carnegie-Rochester Conference Series on Public Policies, 39: 195-214.

- (2002): "A Half-Century of Changes in Monetary Policy". En Conference in Honor of Milton Friedman, University of Chicago, November.

Walsh, C. E. (1995): “Optimal Contracts for Central Bankers”. En American Economic Review, 85 (1), Mar.: 150-167. 